\title{
Identification of pristine and defective graphene nanoribbons by phonon signatures in the electron transport characteristics
}

Christensen, Rasmus Bjerregaard; Frederiksen, Thomas; Brandbyge, Mads

Published in:

Physical Review B

Link to article, DOI:

10.1103/PhysRevB.91.075434

Publication date:

2015

Document Version

Publisher's PDF, also known as Version of record

Link back to DTU Orbit

Citation (APA):

Christensen, R. B., Frederiksen, T., \& Brandbyge, M. (2015). Identification of pristine and defective graphene nanoribbons by phonon signatures in the electron transport characteristics. Physical Review B, 91(7), 075434. https://doi.org/10.1103/PhysRevB.91.075434

\section{General rights}

Copyright and moral rights for the publications made accessible in the public portal are retained by the authors and/or other copyright owners and it is a condition of accessing publications that users recognise and abide by the legal requirements associated with these rights.

- Users may download and print one copy of any publication from the public portal for the purpose of private study or research.

- You may not further distribute the material or use it for any profit-making activity or commercial gain

- You may freely distribute the URL identifying the publication in the public portal 


\title{
Identification of pristine and defective graphene nanoribbons by phonon signatures in the electron transport characteristics
}

\author{
Rasmus B. Christensen, ${ }^{1}$ Thomas Frederiksen, ${ }^{2,3}$ and Mads Brandbyge ${ }^{1, *}$ \\ ${ }^{1}$ Center for Nanostructured Graphene (CNG), Department of Micro- and Nanotechnology, Technical University of Denmark, Ørsteds Plads, \\ Bldg. 345E, DK-2800 Kongens Lyngby, Denmark \\ ${ }^{2}$ Donostia International Physics Center (DIPC), Paseo Manuel de Lardizabal 4, Donostia-San Sebastián, Spain \\ ${ }^{3}$ IKERBASQUE, Basque Foundation for Science, Bilbao, Spain \\ (Received 18 December 2014; revised manuscript received 4 February 2015; published 26 February 2015)
}

\begin{abstract}
Inspired by recent experiments where electron transport was measured across graphene nanoribbons (GNRs) suspended between a metal surface and the tip of a scanning tunneling microscope [Koch et al., Nat. Nanotechnol. 7, 713 (2012)], we present detailed first-principles simulations of inelastic electron tunneling spectroscopy (IETS) of long pristine and defective armchair and zigzag nanoribbons under a range of charge carrier conditions. For the armchair ribbons we find two robust IETS signals around 169 and $196 \mathrm{mV}$ corresponding to the $D$ and $G$ modes of Raman spectroscopy as well as additional fingerprints due to various types of defects in the edge passivation. For the zigzag ribbons we show that the spin state strongly influences the spectrum and thus propose IETS as an indirect proof of spin polarization.
\end{abstract}

DOI: 10.1103/PhysRevB.91.075434

PACS number(s): 81.05.ue, 73.63.-b, 72.10.Di

\section{INTRODUCTION}

Graphene as the basis of a new generation of electronics $[1,2]$ has been the center of much attention in the last years, and devices based on nanostructured graphene have been put forward. The most generic form of nanostructured graphene is graphene nanoribbons (GNRs) [3], and other structures, such as graphene antidot lattices [4,5], can be viewed as networks of them. GNRs are potential candidates for molecular wires with tailored conductance properties. For graphene-based nanostructures the edges and their passivation, as well as defects inside the structure, can play crucial roles for the transport properties [6]. However, characterization of edge passivation or structural/chemical defects is challenging especially after device fabrication. Raman spectroscopy [7] can give information about defects on large areas of the sample, while tip-enhanced Raman spectroscopy (TERS) [8] have been used in combination with STM on GNRs. However, Raman studies involve averages over larger areas $(>10 \mathrm{~nm})$, and does not yield information about the impact of vibrations on transport. In that aspect inelastic electron tunneling spectroscopy (IETS) serves as a way of performing nondestructive characterization yielding vibrational/phonon fingerprints of a range of defects. In order to interpret IETS experiments, theoretical modeling of the inelastic signals in the electronic current due to electron-phonon (e-ph) scattering is needed.

GNRs have been fabricated using different strategies including lithographic techniques [9], chemical synthesis [10,11], epitaxial growth [12], and longitudinal unzipping of carbon nanotubes [13]. Furthermore, several groups have succeeded in atomically precise bottom-up fabrication of armchair GNRs (AGNR) [14,15], chiral GNRs [16], and AGNR heterojunctions [17] grown on metal surfaces. Experimentally, the vibrational properties have been investigated by Raman spectroscopy and the electronic structure has been mapped out by STM, angle-resolved (two-photon) photoemission and

*mads.brandbyge@ nanotech.dtu.dk high-resolution electron energy loss spectroscopy $[8,18,19]$. Signatures of phonon excitation were observed by STM in the differential conductance spectroscopy performed at the zigzag termini state of AGNRs adsorbed on $\mathrm{Au}(111)$, and these signatures were shown to be sensitive to modifications in the local atomic geometry [20]. AGNRs have also been lifted up from the weakly bonding $\mathrm{Au}(111)$ surface with the tip of a STM enabling measurements of the voltage-dependent conductance in suspended configurations [21].

From the theoretical side density-functional theory (DFT) has been used to investigate the stability of structural and chemical reconstructions of GNR edges [22-24], together with the transport and band-gap engineering [6,25-28]. The vibrational properties and phonon band structure have been calculated with empirical potentials [29] and DFT [30,31]. In addition, there have been theoretical predictions $[32,33]$ of the Raman spectrum, in good agreement with experiments [14,34]. For a finite AGNR the role of zigzag termini states have been studied theoretically, comparing DFT to the many-body Hubbard model [35].

Inspired by the recent lifting experiments by Koch et al. [21], we here investigate theoretically the signals of e-ph scattering in the conductance of long GNRs between metal electrodes. Our aim is twofold. First, we want to address the role phonon scattering in the transport characteristics of pristine GNRs. Second, we wish to compute detailed IETS for different GNRs under varying charge carrier concentrations and explore how different types of realistic defects may modify the IETS and thus possibly be directly probed in transport measurements. We focus on the two most generic edge types, namely armchair (AGNR) and zigzag (ZGNR), and pay attention to the effects of spin polarization in the latter case. In actual experiments the substrate or an applied gate potential control the Fermi level $E_{F}$ in the ribbons. To address this variability we scan $E_{F}$ using a numerically effective scheme enabling fast calculations of the IETS [36]. We find that the AGNR generally display two robust IETS signals around 169 and $196 \mathrm{mV}$ corresponding to the $D$ and $G$ modes of Raman spectroscopy and that a dehydrogenated dimer at the edge 


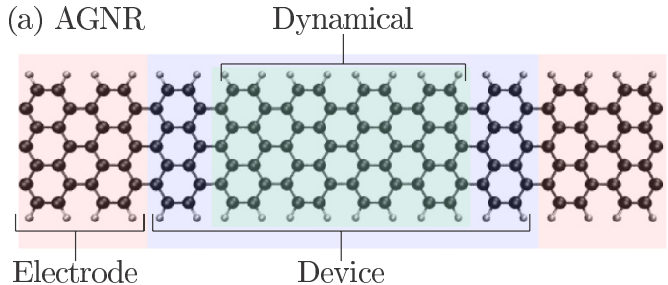

(b)

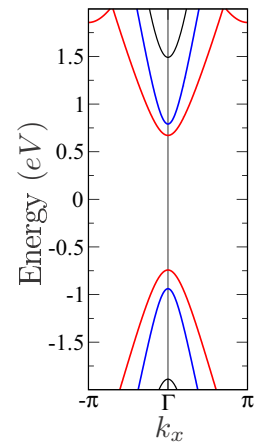

(c)

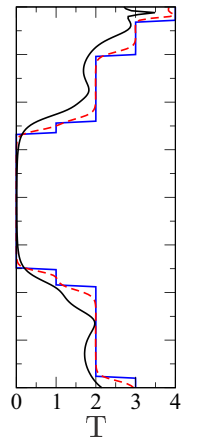

(d)

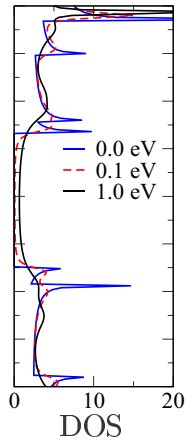

(e) ZGNR

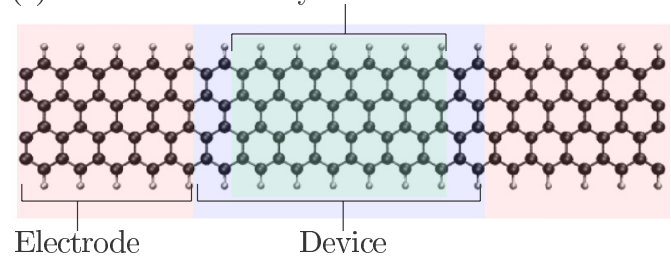

(f)

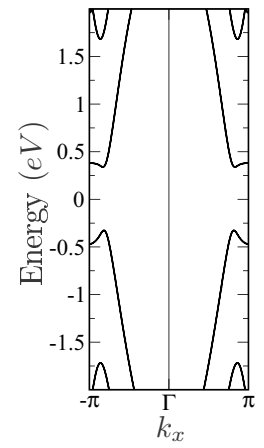

(g)

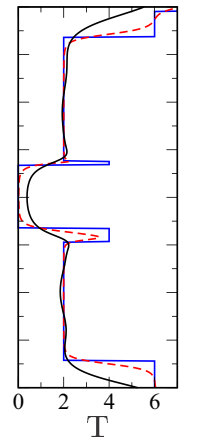

(h)

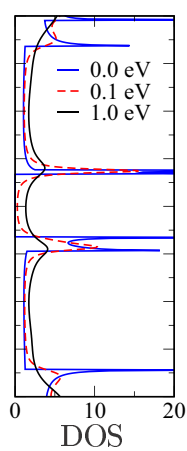

FIG. 1. (Color online) (a) Computational setup for a pristine AGNR showing electrode, device, and dynamical regions. (b) Electronic band structure ( $k_{x}$ is in units of inverse unit cell length). The different bands are colored according to symmetry of the electronic states. Red: symmetric, corresponding to Figs. 2(a) and 2(b). Blue: antisymmetric, corresponding to Figs. 2(c) and 2(d). (c) Electronic transmission for varying electrode broadening describing the coupling to the metal contacts, $\eta=0,0.1,1 \mathrm{eV}$, see text. (d) Electronic DOS projected onto the dynamical region. (e)-(h) Similar entities for the pristine ZGNR case.

should further leave a clear defect signal at around $245 \mathrm{mV}$. For the ZGNR we find that the spin polarization breaks the mirror symmetry around the middle of the ribbon resulting in IETS signals from a range of modes around the $D$ - and $G$-mode energies. Both AGNR and ZNGR defects, which break the planar symmetry of ribbons, allow for contributions to the IETS from out-of-plane phonon modes.

The paper is organized as follows. First we discuss our atomistic model setup for the density functional and electron transport calculations, and outline the approach for the IETS simulations. In Sec. III we present our results for pristine AGNR and ZGNR and relate their transport properties and IETS to the band structures. In Sec. IV we turn to the defective systems by considering realistic possibilities of defects in the edge passivation, backbone bonding motifs, and presence of adatoms. Finally, a summary and our conclusions are presented in Sec. V.

\section{METHODS}

We calculate the electronic and vibrational structure from DFT using the academic codes SIESTA/TRANSIESTA [37,38]. We employ the generalized gradient approximation (GGA) for the exchange-correlation functional [39], a single- $\zeta$ polarized (SZP) basis set for the carbon and hydrogen atoms, and use a cut-off energy of 400-500 Ry for the real-space grid. These choices, balancing accuracy and computational cost, provide a good description to investigate trends and general behavior of the substantial number of systems considered in this work.

The vibrational degrees of freedom, calculated by diagonalization of the dynamical matrix, and the e-ph couplings are extracted from finite differences as implemented in the
INELASTICA code [40-42]. The armchair and zigzag GNRs considered here are shown in Fig. 1. We adopt the usual two-probe setup with the device region $(D)$ coupled to left $(L)$ and right $(R)$ electrodes with all electronic matrix elements expressed in a local basis set. The primitive unit cell of the AGNR (ZGNR) consists of 18 (10) atoms and in our calculations this unit cell is repeated 10 (18) times in the transport direction to form the scattering regions illustrated in Figs. 1(a) and 1(e). The electrode couplings $\boldsymbol{\Gamma}_{L / R}$ are included on the two first/last unit cells before folding onto $D$. In our treatment a subset of atoms in $D$ is allowed to vibrate. We fix this dynamical region, restricted by the condition that the e-ph couplings are fully included inside $D$, to the four and six central unit cells for the AGNR and ZGNR, respectively. The corresponding e-ph couplings used to calculate the inelastic electron transport are thus expressed in the center six unit cells for the AGNR and eight unit cells for the ZGNR. The convergence of our results with the size of the dynamical region is addressed below.

We generally consider nanoribbons that are suspended between two metallic leads. In the case of the lifting experiments [21], these would correspond to the metal sample surface and the STM tip. Here we wish to focus on the action inside the GNRs and put aside the possible complications due to the detailed electronic structure of the metals, and the metal-GNR interface in particular. To this end we introduce a simple model of the metal electrodes without substantial electronic features: we use semi-infinite GNRs with highly broadened states (effectively smearing out energy gaps). In practice this is done by adding a finite numerical imaginary part $\eta$ to the energy argument in the electrode recursion calculation [43]. This scheme ensures that the phonon effects 


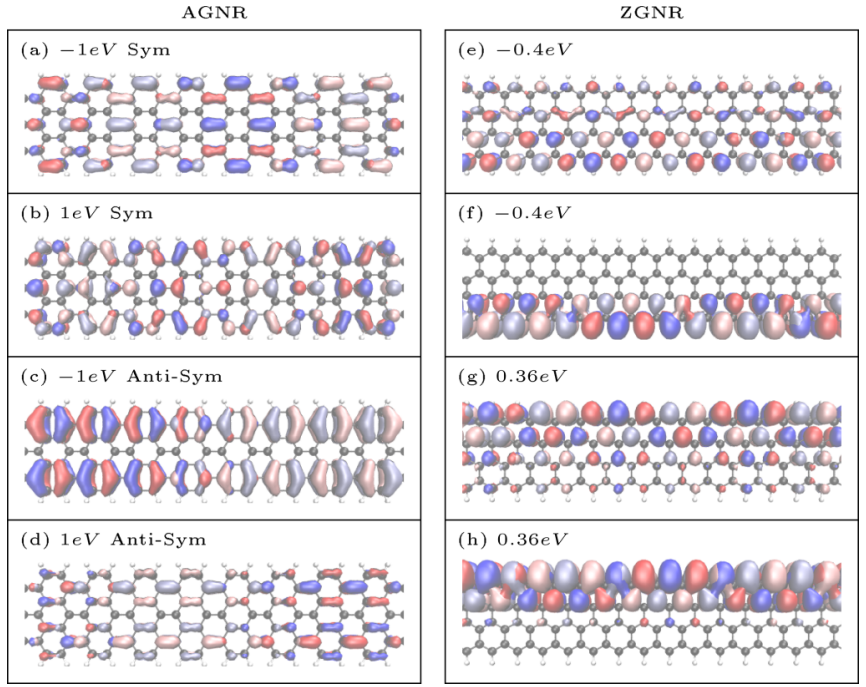

FIG. 2. (Color online) (a)-(d) Electron transmission eigenchannels for the clean AGNR for the valence bands at $E-E_{F}=-1 \mathrm{eV}$ and for the conduction bands at $E-E_{F}=1 \mathrm{eV}$. (e)-(h) Electron transmission eigenchannels for the clean ZGNR in the valence bands at $E-E_{F}=-0.4 \mathrm{eV}$ and in the conduction bands at $E-E_{F}=$ $0.4 \mathrm{eV}$ for one spin component. The eigenchannels for the other spin component are simply mirror images around the middle of the ZGNR (not shown). The red/blue (pink/gray) isosurfaces represent the real (imaginary) part and sign of the scattering state wave function. For all eigenchannel calculations the electrode broadening was set to zero $(\eta=0 \mathrm{eV})$.

originate from the GNRs themselves and not from details of the metal-GNR interface, which is generally unknown in the STM experiments. The electronic band structures for the infinite ribbons, along with the transmission and density of states (DOS) for $\eta=0,0.1,1 \mathrm{eV}$ are shown in Figs. 1(b), 1(c), and 1(d) and Figs. 1(f), 1(g), and 1(h) for AGNR and ZGNR, respectively. We note that the broadened transmission spectrum [Fig. 1(d)] is quite consistent with the experimentally reported differential conductance curves for AGNR [21]. The electronic states involved in the transport are shown in Fig. 2 in terms of the transmission eigenchannels [44] in the valence and conduction bands of the AGNR and ZGNR. Their spatial symmetry play a significant role for the selection rules involved in the inelastic scattering as discussed later.

In principle, the electronic structure should be evaluated at finite bias. However, without a detailed model of the connection to the metal electrodes (where an important part of the voltage drop will take place) and for sufficiently long systems (in which the electric field will be small), it is reasonable to use the zero-voltage electronic structure and to simply assume a symmetric voltage drop over the two identical, idealized device-electrode interfaces. More specifically, in the following we will treat the applied potentials non-self-consistently. An equal voltage drop $(V)$ is used at the left and right electrodes for these completely (or nearly) left-right symmetric structures, and shift the electronic states and chemical potentials in the left/right electrodes by $\pm V / 2$. We introduce the applied gate voltage $V_{G}$ (mimicking actual doping or electrostatic gating that modify the charge carrier concentration in an experimental setup) as a shift in all energies. Thus close to the excitation threshold of a phonon $\lambda$ we consider inelastic scattering between states at energies around the chemical potentials $\mu_{L / R}=E_{F} \pm \hbar \omega_{\lambda} / 2+V_{G}$.

\section{A. Computational scheme for IETS}

For a device strongly coupled to the electrodes, a coupling between the electron current $I(V)$ and a phonon mode $\lambda$ ideally shows up at zero temperature as a step discontinuity in the differential conductance when the inelastic phonon emission process becomes energetically allowed, that is, when the chemical potential difference exceeds the quantum of vibrational energy $\left|\mu_{L}-\mu_{R}\right|=\hbar \omega_{\lambda}$. Thus, around the emission threshold the electronic states involved in the scattering process are those at $\mu_{L}$ and $\mu_{R}$. The IETS signal, conventionally expressed as the ratio between the second and first derivatives of the current with respect to the voltage,

$$
\text { IETS }=\frac{\partial_{V}^{2} I(V)}{\partial_{V} I(V)},
$$

is calculated by considering the e-ph coupling as the perturbation on the current, evaluated using the nonequilibrium Green's functions (NEGF). In the so-called lowest order expansion (LOE) the inelastic part of the differential conductance can be written as [36]

$$
\begin{aligned}
\partial_{V} I(V)= & \gamma_{\lambda} \partial_{V} \mathcal{I}^{\operatorname{sym}}\left(V, \hbar \omega_{\lambda}, T\right) \\
& +\kappa_{\lambda} \partial_{V} \mathcal{I}^{\operatorname{asym}}\left(V, \hbar \omega_{\lambda}, T\right),
\end{aligned}
$$

where summation over the vibration index $\lambda$ is assumed. $\mathcal{I}^{\text {sym }}$ and $\mathcal{I}^{\text {asym }}$ are the "universal" (system-independent) functions that depend on the applied bias $V$, phonon energy $\hbar \omega_{\lambda}$, and the temperature $T$. Assuming the electronic and phononic distribution functions are given by the Fermi-Dirac and Bose-Einstein distributions, respectively, their analytical expressions can be written as

$$
\begin{aligned}
\mathcal{I}^{\text {sym }} \equiv & \frac{\mathrm{G}_{0}}{2 e} \sum_{s= \pm} s\left(\hbar \omega_{\lambda}+s e V\right) \\
& \times\left(\operatorname{coth} \frac{\hbar \omega_{\lambda}}{2 k_{B} T}-\operatorname{coth} \frac{\hbar \omega_{\lambda}+s e V}{2 k_{B} T}\right), \\
\mathcal{I}^{\text {asym }} \equiv & \frac{\mathrm{G}_{0}}{2 e} \int_{-\infty}^{+\infty} d \varepsilon \mathcal{H}\left\{f\left(\varepsilon_{-}^{\prime}\right)-f\left(\varepsilon_{+}^{\prime}\right)\right\}(\varepsilon) \\
& \times[f(\varepsilon-e V)-f(\varepsilon)],
\end{aligned}
$$

where $\mathrm{G}_{0}=2 e^{2} / h$ is the conductance quantum, $f(\varepsilon)$ is the Fermi-Dirac function, $\varepsilon_{s}^{\prime} \equiv \varepsilon^{\prime}+s \hbar \omega_{\lambda}$, and $\mathcal{H}$ denotes the Hilbert transform.

The signal amplitudes $\gamma_{\lambda}$ and $\kappa_{\lambda}$ of the symmetric and antisymmetric signals in the differential conductance are even and odd in bias, respectively. For a symmetric structure the asymmetric signal vanishes in the wide-band approximation (LOE-WBA) [40]. However, this is not guaranteed in the more general treatment employed here [36], where the energy dependence of the electronic structure is explicitly taken into account. The amplitudes $\gamma_{\lambda}$ and $\kappa_{\lambda}$ are expressed in terms of 
electronic structure quantities and e-ph couplings [36],

$$
\begin{gathered}
\gamma_{\lambda}=\operatorname{Tr}\left[\mathbf{M}_{\lambda} \tilde{\mathbf{A}}_{L}\left(\mu_{L}\right) \mathbf{M}_{\lambda} \mathbf{A}_{R}\left(\mu_{R}\right)\right]+\operatorname{Im} B_{\lambda}, \\
\kappa_{\lambda}=2 \operatorname{Re} B_{\lambda},
\end{gathered}
$$

where $B_{\lambda}$ is defined as

$$
\begin{aligned}
B_{\lambda} \equiv & \operatorname{Tr}\left[\mathbf{M}_{\lambda} \mathbf{A}_{R}\left(\mu_{L}\right) \boldsymbol{\Gamma}_{L}\left(\mu_{L}\right) \mathbf{G}^{r}\left(\mu_{L}\right) \mathbf{M}_{\lambda} \mathbf{A}_{R}\left(\mu_{R}\right)\right. \\
& \left.-\mathbf{M}_{\lambda} \mathbf{G}^{a}\left(\mu_{R}\right) \boldsymbol{\Gamma}_{L}\left(\mu_{R}\right) \mathbf{A}_{R}\left(\mu_{R}\right) \mathbf{M}_{\lambda} \mathbf{A}_{L}\left(\mu_{L}\right)\right] .
\end{aligned}
$$

In the above, $\mathbf{M}_{\lambda}$ denotes the e-ph coupling matrix for mode $\lambda, \mathbf{G}^{r / a}$ are the retarded/advanced unperturbed Green's functions, and $\mathbf{A}_{\alpha}=\mathbf{G}^{r} \boldsymbol{\Gamma}_{\alpha} \mathbf{G}^{a}$ are the spectral density matrices for left/right moving states with the time-reversed version $\tilde{\mathbf{A}}_{\alpha}=\mathbf{G}^{a} \boldsymbol{\Gamma}_{\alpha} \mathbf{G}^{r}$. The purely electronic quantities are thus being evaluated at the chemical potentials of the left/right electrodes corresponding to the excitation threshold for each vibration. We compute $\mathbf{M}_{\lambda}$ with the finite-difference scheme of INELASTICA taking the vacuum energy as a common reference (in absence of real metal leads to pin the Fermi energy) [41].

In the localized atomic basis set of SIESTA all the above quantities are matrices defined in the electronic space corresponding to region $D$. The second derivatives of the universal functions in Eqs. (3) and (4) are sharply peaked around the phonon threshold. For this reason the coefficients $\gamma_{\lambda}$ and $\kappa_{\lambda}$ can be considered voltage independent with their values computed exactly at the threshold. Due to the computational efficiency of the LOE scheme described above we are able to evaluate the IETS on a fine grid of gate voltages $V_{G}$ spanning a large range of relevant values between valence and conduction bands of the GNRs.

\section{PRISTINE GRAPHENE NANORIBBONS}

We first turn to the IETS results of the two pristine (clean) ribbons, and in the following section to the impact of selected defects in the IETS. As our main system we focus on the AGNR systems directly relevant for the lifting experiments [21]. The results for the ZGNR are provided mainly as comparison and to look into the role of chirality and in particular effects rooted in spin polarization, and thus we discuss these separately.

\section{A. Pristine armchair nanoribbons}

As representative of the AGNR class we have investigated a pristine AGNR with a width of $W=7$ dimers (7-AGNR) corresponding to a C-C edge distance of $7.5 \AA$ (see Fig. 1). It presents a direct semiconducting band gap $E_{g}$ due to the lateral confinement and can be classified as "large-gap ribbons" since $p=2$ is an integer in the relation $W=3 p+1$ [1]. We obtain $E_{g} \approx 1.3 \mathrm{eV}$ at the present level of approximation (DFT-GGA and SZP basis set), as seen from the electronic band structure shown in Fig. 1(b). This value is smaller than those estimated experimentally $\left(E_{g} \approx 2.3-2.6 \mathrm{eV}\right.$ for a flat AGNR on $\mathrm{Au}(111)[19,45]$ and $E_{g} \approx 2.7 \mathrm{eV}$ for an AGNR suspended between surface and STM tip [21]) due to the underestimation of electron-electron interaction [46] which plays an more important role in quasi-one-dimensional GNRs compared to pristine graphene. Dielectric screening from the substrate also influences significantly the actual gap size: a band gap of $3.2 \mathrm{eV}$ for a 7-AGNR was found to be lowered to

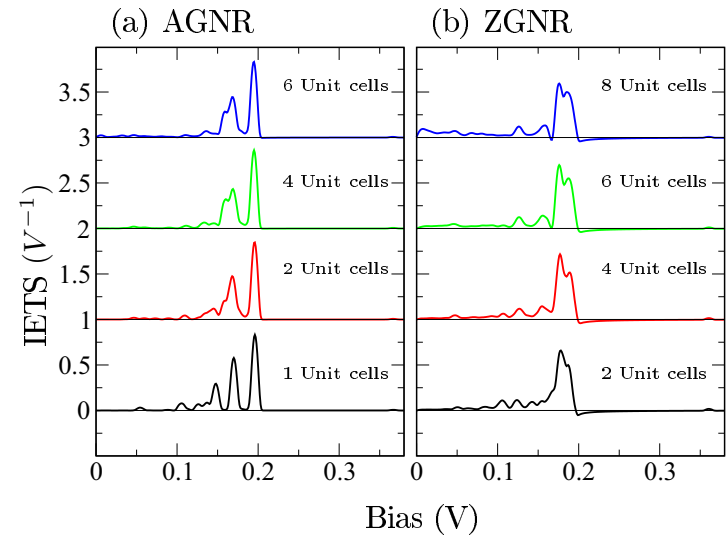

FIG. 3. (Color online) Convergence of the intrinsic IETS for pristine (a) AGNR and (b) ZGNR as a function of the size of the dynamical region (stated in the legends). The results (offset for clarity) are normalized with respect to the number of vibrating unit cells, i.e., we show the IETS amplitude per $\mathrm{H}_{4} \mathrm{C}_{14}$ segment for AGNR and per $\mathrm{H}_{2} \mathrm{C}_{8}$ segment for ZGNR. No gate voltage is applied $\left(V_{G}=0.0 \mathrm{~V}\right)$.

$2.7 \mathrm{eV}$ on a hexagonal boron-nitride $(\mathrm{hBN})$ substrate using $G W$ calculations [47], similar to the lowering calculated for a 7-AGNR on $\mathrm{Au}(111)$ [48]. In general we expect that underestimation of band gaps would mainly amount to a simple scaling of the Fermi level position within the gap.

We first discuss the effect of the finite size of the dynamical region in our treatment. Figure 3(a) shows how the IETS signals for the AGNR (at fixed gate voltage $V_{G}=0.0 \mathrm{~V}$ ) vary as a function of the size of the dynamical region, ranging from one to six unit cells. For easy comparison, the data are normalized by the number of vibrating unit cells. As the signal amplitudes in this representation are roughly constant, we conclude that the absolute IETS simply scale linearly with the active e-ph coupling region. Consequently, the magnitudes in IETS may thus provide insight into the active scattering region in actual experiments. Furthermore, as we find that both IETS amplitude and shape is well converged with four vibrating unit cells, we fix the dynamical region to this size in the following analysis.

The computed IETS signals for the AGNR as a function of varying gate voltage are shown in Fig. 4(a) as a density plot. Specific IETS spectra at selected gate voltages are shown in Fig. 4(c) for both the intrinsic part (temperature broadening at $T=4.2 \mathrm{~K}$ ) as well as that one would observe employing the experimental lock-in technique (additional broadening due to a modulation voltage of $V_{\mathrm{rms}}=5 \mathrm{mV}$ ). We find that for the AGNR there are generally two well-defined IETS signals appearing around 169 and $196 \mathrm{meV}$, corresponding to the $D$ (ring breathing) and $G$ ( $E_{2 g}$ phonon) modes, respectively, also observed in Raman spectroscopy [7,9]. The $D$ signal also has a shoulder with a local maximum at $159 \mathrm{meV}$ with contributions from several modes. These three distinct features are indicated with vertical lines in Figs. 4(a) and 4(c). Shifting $E_{F}$ inside the gap region with a relatively small gate voltage $\left|V_{G}\right| \lesssim$ $0.5 \mathrm{~V}$ does not affect the IETS appreciably. However, when $E_{F}$ comes close to the conduction band of the AGNR the signal increases by a factor of 5 and a small peak-dip feature appears similar to the one reported for gated benzene-dithiol molecular 

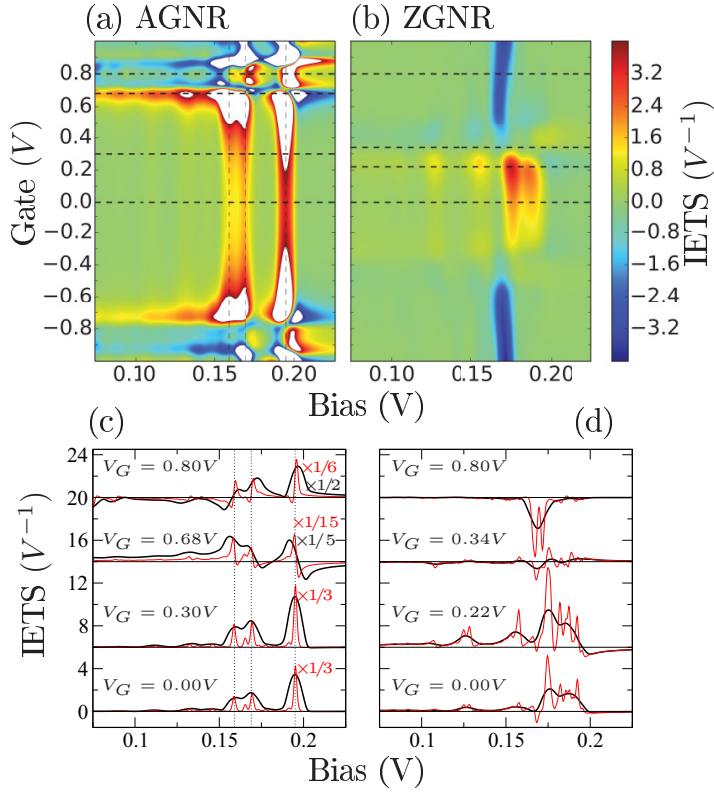

FIG. 4. (Color online) IETS signals as a function of gate voltage for (a) pristine AGNR (four vibrating unit cells) and (b) pristine ZGNR (six vibrating unit cells). Vertical dashed lines are guides to the eye indicating the energy of the most contributing vibrational modes. Specific IETS signals (offset for clarity) for the (c) AGNR and (d) ZGNR at selected gate voltages marked with horizontal dashed lines in (a) and (b). Broadening originates from temperature $T=$ $4.2 \mathrm{~K}$ and a lock-in modulation voltage $V_{\text {rms }}=5 \mathrm{mV}$ (except for the thin red lines in the lower panels with $V_{\mathrm{rms}}=0 \mathrm{mV}$ ).

contacts [36,49]. Upon further gating into the conduction band the IETS signals undergo a sign reversal (from peaks to dips) as the transmission increases beyond approximately 0.5 for the involved channels [50]. This sign reversal can be traced back to the competition between inelastic and elastic scattering processes [51-53]. Similar effects are also found by gating into the valence band of the AGNR.

We can easily identify the most important vibrational mode vectors $v_{\lambda}$ for the IETS from the two amplitudes $\left|\gamma_{\lambda}\right|$ and $\left|\kappa_{\lambda}\right|$ given in Eqs. (5) and (6). These modes can be further analyzed in terms of the phonons in the infinite AGNR. To do so we introduce the measure $F_{n k}$ representing the overlap between modes in the finite dynamical cell and the phonon band modes weighted by the size of the IETS signal,

$$
F_{n k}\left(V_{G}\right)=\sum_{\lambda}\left|\gamma_{\lambda}\left(V_{G}\right)\right|\left|u_{n k}\left(1, e^{i k}, \ldots, e^{i(N-1) k}\right) v_{\lambda}\right|^{2},
$$

where $u_{n k}$ is the phonon band mode indexed by $n$, and $v_{\lambda}$ is the modes in a finite $N$ primitive cell long dynamical region index by $\lambda$.

The projections $F_{n k}\left(V_{G}=0 \mathrm{~V}\right)$ are depicted as widths of the phonon bands in Fig. 5(a), where the red, green, and blue colors refer to modes with frequencies in the ranges $\hbar \omega>180 \mathrm{meV}, 180>\hbar \omega>162 \mathrm{meV}$, and $\hbar \omega<162 \mathrm{meV}$, respectively. In total four bands contribute to the IETS signal corresponding to the four signals seen in the intrinsic part of the IETS spectrum in Fig. 4(c). The corresponding $\Gamma$-point phonon modes inside the primitive cell for the infinite ribbon are shown in Figs. 5(b)- 5(e).

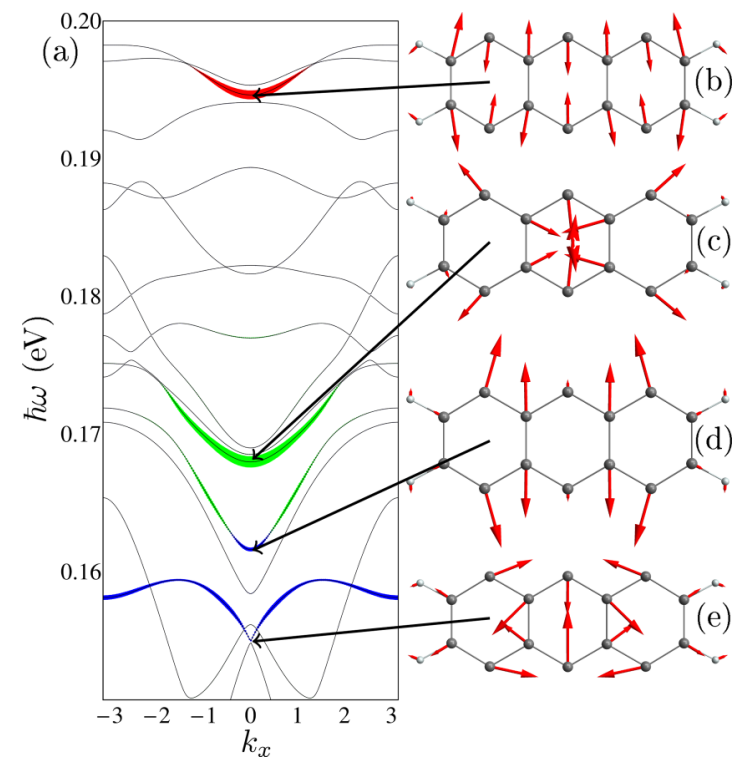

FIG. 5. (Color online) (a) Computed phonon band structure for the pristine, infinite AGNR ( $k_{x}$ is in units of inverse unit cell length). The magnitude of the red, green, and blue bands [corresponding to the three vertical lines in Fig. 4(c)], is proportional to the signal size weighted overlap $\left[F_{n k}\left(V_{G}=0 \mathrm{~V}\right)\right.$ in Eq. (8)], between the repeated band vector and modes with frequencies $\hbar \omega>180 \mathrm{meV}, 180>\hbar \omega>$ $162 \mathrm{meV}$, and $\hbar \omega<162 \mathrm{meV}$ for red, green, and blue, respectively. The red band is scaled by 0.2 compared to blue and green. (b)-(e) Selected phonon band modes at $\Gamma$ for the infinite structure which, according to the projection, characterize the active IETS modes.

In summary, the $G$ signal is due to the highest optical longitudinal mode shown in Fig. 5(b), corresponding to the red band in Fig. 5(a). The $D$ signal originates primarily from the ring breathing mode shown in Fig. 5(c), and has a small contribution from a longitudinal mode shown in Fig. 5(e) corresponding to the green bands in Fig. 5(a), while the shoulder in the $D$-band signal is due to the edge-phonon mode shown in Fig. 5(e) corresponding to the blue band in in Fig. 5(a).

\section{B. Pristine zigzag nanoribbon}

We next turn to our results for the pristine ZGNR shown in Fig. 1(e). It has a width of $W=4$ zigzag "chains" (4-ZGNR) corresponding to a C-C edge distance of $7.26 \AA$. The breaking of sublattice symmetry for the ZGNR and lack of pseudophase result in different selection rules for the matrix elements and difference in for example Raman signals [33]. The ZGNR generally presents spin-polarized edge states exhibiting a small band gap at the DFT level [1], in our case $E_{g} \approx 0.6 \mathrm{eV}$ (we note that this gap disappears in simpler tight-binding descriptions [1] or spin-degenerate DFT calculations). The spinpolarized edge states play the major role for the conduction, see the spin-down eigenchannels visualized in Figs. 2(e)-2(h).

Since the edge states break the mirror symmetry with respect to the middle of the ribbon, there are fewer symmetryforbidden inelastic transitions between the scattering states for the ZGNR. Thus, we expect a wider range of modes to contribute to the IETS signal as compared to the AGNR 


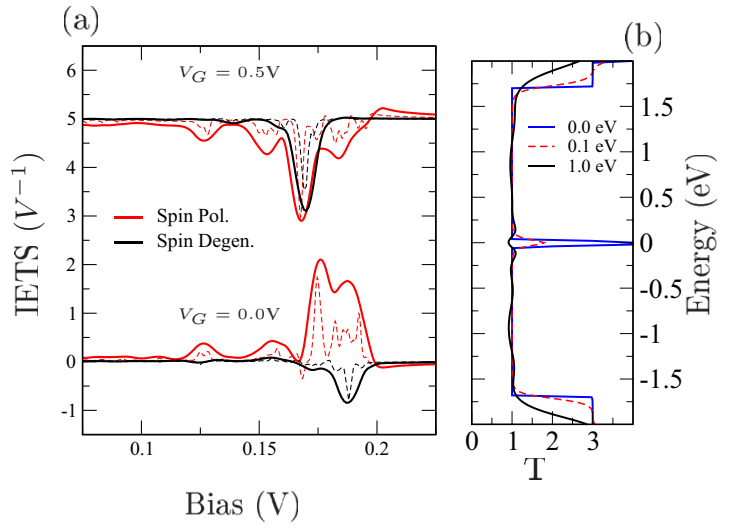

FIG. 6. (Color online) (a) IETS signals with $V_{G}=0.0$ and $0.5 \mathrm{~V}$ (offset for clarity) for the pristine ZGNR (six vibrating unit cells). The black lines correspond to spin-degenerate calculations while the red lines are the spin-up components of spin-polarized calculations. Broadening originates from temperature $T=4.2 \mathrm{~K}$ and modulation voltage $V_{\mathrm{rms}}=5 \mathrm{mV}$ (full lines) or $V_{\mathrm{rms}}=0 \mathrm{mV}$ (dashed lines). (b) Electronic transmission from spin-degenerate calculations with varying electrode broadening describing the coupling to the metal contacts, $\eta=0,0.1,1 \mathrm{eV}$ [see also Fig. 1 (g) for the corresponding spin-polarized case].

case. Indeed this is in agreement with the findings shown in Figs. 3(b), 4(b), and 4(d). The greater number of modes contributing to the IETS for the ZGNR results in broader signals with similar magnitudes as compared to the IETS for AGNR. As for the AGNR case the IETS signal is well converged with a dynamical region consisting of six vibrating unit cells [Fig. 3(b)].

For ZGNRs scattering against the ring breathing mode is forbidden by symmetry, thus the IETS is generally characterized by transverse and longitudinal modes. To explore the impact of spin polarization on the ZGNR-IETS we compare in Fig. 6 the results from both spin-degenerate and spin-polarized calculations. Without gate voltage $\left(V_{G}=\right.$ $0 \mathrm{~V})$ the IETS display opposite signs due to the spininduced gap. Only a single peak contributes to the spindegenerate IETS while several peaks contribute to the spinpolarized IETS. Even if the ZGNR is tuned by $V_{G}=$ $0.5 \mathrm{~V}$ to become metallic and the two treatments then show the same overall sign in IETS, the spin-polarized IETS persists to show a much richer structure. This difference suggests that IETS could be a way to indirectly observe spin-polarized edge states.

Projecting the modes contributing to the IETS onto the phonon band modes further underlines how several bands with different symmetries contribute to the spin-polarized IETS, while only a couple of bands contributes to the spin-degenerate IETS, see Fig. 7. Again we use Eq. (8) for this characterization, where the overlap for $V_{G}=0.0 \mathrm{~V}$ corresponds to the red color and the overlap for $V_{G}=0.5 \mathrm{~V}$ corresponds to the difference between the blue and red color in Fig. 7, respectively. It is clear that spin polarization permits more modes to contribute to the IETS. In contrast to the spin-degenerate case, where the symmetric electronic states (with respect to the middle of the ribbon) only can couple to the symmetric vibration modes, the

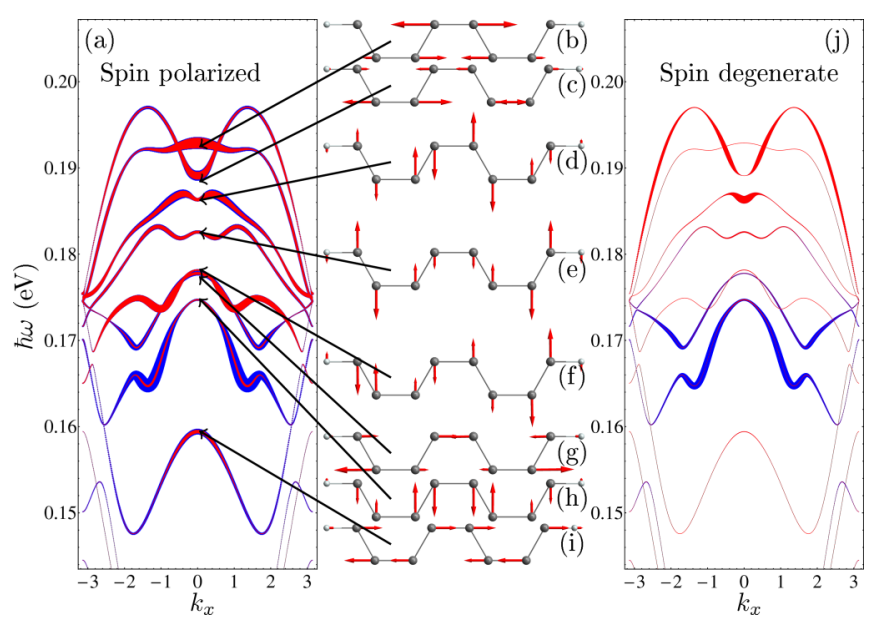

FIG. 7. (Color online) The phonon band structure of the ZGNR ( $k_{x}$ is in units of inverse unit cell length), together with the $\Gamma$-point modes. The widths of the red bands are proportional to the weight function $F(0 \mathrm{~V})$ [Eq. (8)], while the widths of the blue bands are proportional to $F(0 \mathrm{~V})+F(0.5 \mathrm{~V})$.

symmetry lowering of the electronic states by spin polarization opens up also for scattering also via odd modes.

\section{DEFECTIVE GRAPHENE NANORIBBONS}

In this section we address the modification and new signals in IETS that arise due to various defects in the GNR. Regardless of the fabrication method, defects will inevitably occur. For example, if the AGNRs are synthesized from a precursor molecule, involving heating and dehydrogenation, as reported by Cai et al. [14] and Blankenburg et al. [15], there is a chance that the reaction is incomplete and some of the $\mathrm{C}-\mathrm{C}$ bonds between the precursor molecules do not form. Also there is a chance that a part of the final AGNR will have dehydrogenated edges or are passivated by two hydrogen atoms. Finally, defects may be introduced on purpose by locally dosing a high current from the tip of a STM [20].

\section{A. Defects in AGNRs}

In Fig. 8 we show the structures of pristine AGNR along with eight different defect configurations which we have considered. These include four defects in the edge passivation as follows: A single edge side with an extra hydrogen atom [1H-edge, Fig. 8(b)], two edge sides with each an extra hydrogen atom [2H-edge, Fig. 8(c)], one hydrogen replaced by a fluorine atom [1F-edge, Fig. 8(d)], and a dehydrogenated edge with four hydrogen atoms removed from each side [8H-free, Fig. 8(e)]. We have also considered defects in the atomic structure in the form of one, two, or four broken $\mathrm{C}-\mathrm{C}$ bonds [1C-broken, 2C-broken, 4C-broken, Figs. 8(f)-8(h)] as well as a $\mathrm{Cu}$ adatom on the AGNR [Cu-adatom, Fig. 8(i)]. For all these systems the entire dynamical region was relaxed, i.e., the parts of the AGNRs shown in Fig. 8.

Defects may influence the IETS signal in two ways. First, a defect can have a direct impact by changing the vibrational degrees of freedom. In order for the change in the vibrational spectrum to give a signal in the IETS, the new vibrations 


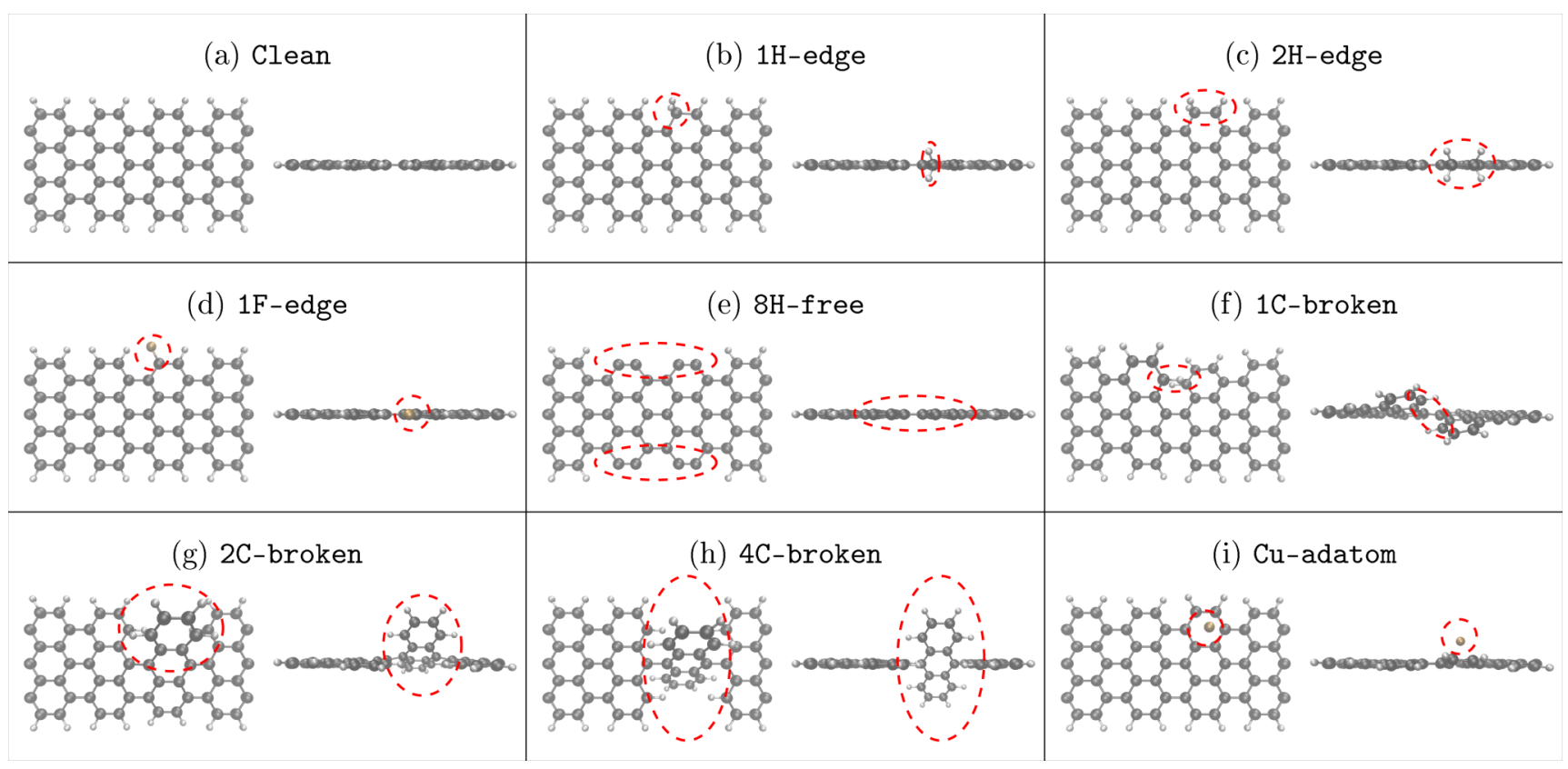

FIG. 8. (Color online) Top and side views of the dynamical region describing the various AGNR defect structures. The dashed red ellipses are guides to the eye highlighting the defect position. (a) Pristine AGNR. (b) One extra H atom on one of the edges. (c) Two extra H atoms on one of the edges. (d) One $\mathrm{H}$ atom replaced by a $\mathrm{F}$ atom. (e) Dehydrogenated edge where $4 \mathrm{H}$ atoms have been removed from each side. (f) One broken $\mathrm{C}-\mathrm{C}$ bond. (g) Two broken C-C bonds. (h) Four broken $\mathrm{C}-\mathrm{C}$ bonds. (i) $\mathrm{Cu}$ adatom in a hollow site on the edge.

must couple to the current, and preferably have frequencies which do not coincide with ones already giving IETS signals for the pristine ribbons. Second, a defect can substantially change the electronic structure and thereby have an impact on the e-ph couplings associated with the active modes or even the transmission eigenchannels of the pristine ribbons, e.g., changing a peak in the IETS to a dip (and vice versa) or enhancing asymmetric contributions via Eq. (4).

The electronic properties of the pristine AGNR is shown in Fig. 9(a). The carbon DOS projected to the device region (red curve) reveals a gap as expected from the band structure [Fig. 1(b)], which is significantly broadened from the coupling to the metallic electrodes. The two valence and two conduction bands in the considered energy range naturally explain that the total transmission (black curve) is bound below a value of 2. Furthermore, the ratio $T / T_{1}<2$ (green dashed line), measuring the minimum number of contributing channels where $T_{1}$ is the transmission of the most transmitting eigenchannel, shows that both channels play a role for the transport, at least away from the edges of the direct band gap. Measurements of shot noise may provide insights into this effective number of conductance eigenchannels $[54,55]$. We can now discuss how the different defects modify the electronic properties. From Figs. 9(b)-9(i) we notice that not all defects change the elastic transmission, and furthermore, a change in elastic transmission needs not be unique for a specific defect.

Instead, IETS may provide a additional fingerprint in the current that can be used to identify the type of defect. Figure 10 shows the computed IETS as a function of gate voltage for the eight different defects. As for the clean structure, the two peaks at 169 and $196 \mathrm{meV}$ corresponding to the $D$ and $G$ Raman modes are dominant for a range of gate values for all the structures. Another feature, which is present in all the systems, is the appearance of several signals close to the band onsets. In the following subsections we discuss in more detail
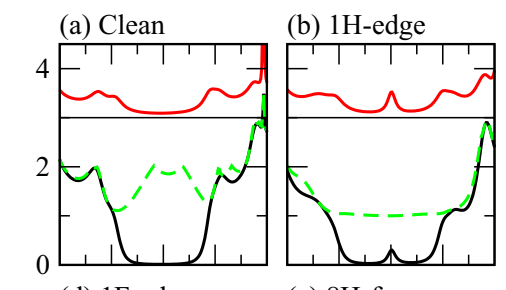

(c) $2 \mathrm{H}$-edge

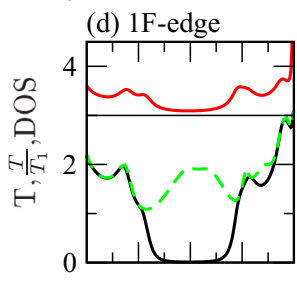

(e) $8 \mathrm{H}$-free
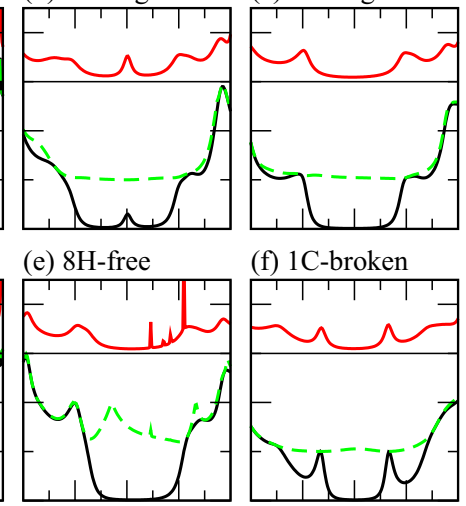

(f) 1C-broken

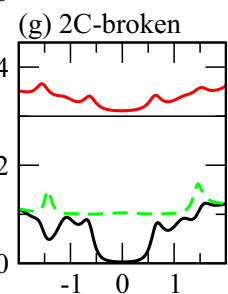

(h) 4C-broken
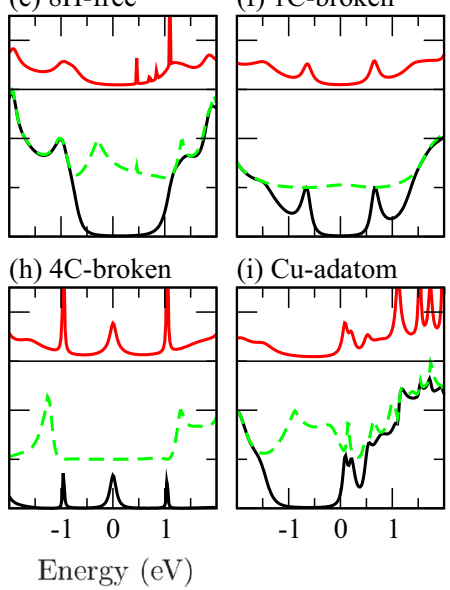

FIG. 9. (Color online) Electronic properties of the AGNR structures shown in Fig. 8. The total transmission is shown with black lines. The ratio $T / T_{1}$, where $T_{1}$ is the transmission originating from the most transmitting eigenchannel, is shown with green dashed lines (this ratio gives a lower bound to the number of contributing eigenchannels). The DOS (arb. units) for the $\mathrm{C}$ atoms in the dynamical region is shown with red lines (offset by three units). 


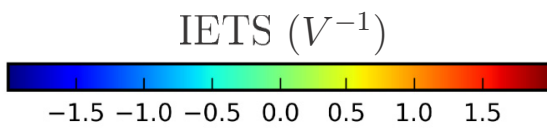

(a) Clean

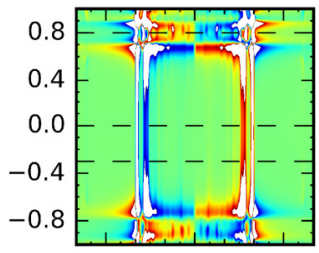

(d) 1F-edge

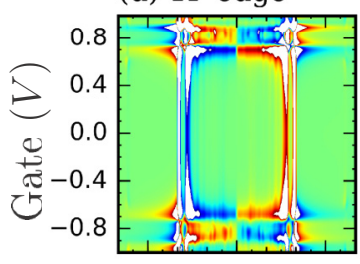

(g) 2C-broken

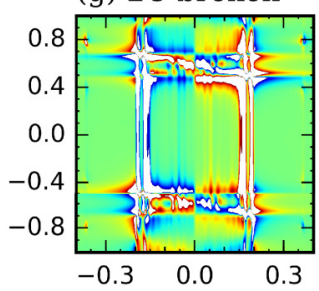

(b) $1 \mathrm{H}$-edge

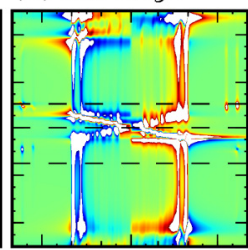

(e) $8 \mathrm{H}$-free

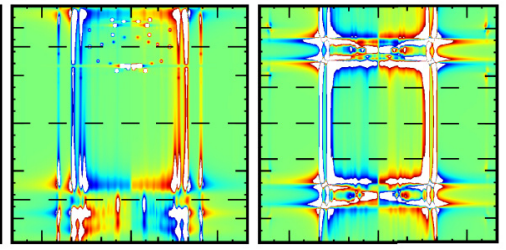

(h) 4C-broken

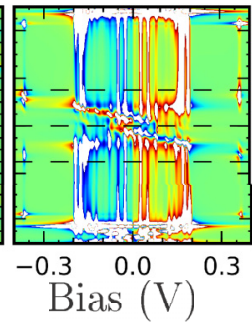

(c) $2 \mathrm{H}$-edge

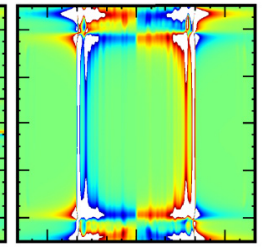

(f) 1C-broken
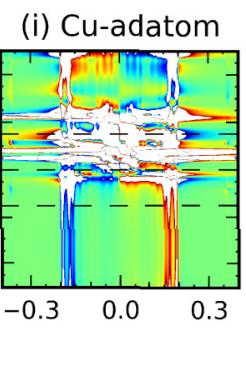
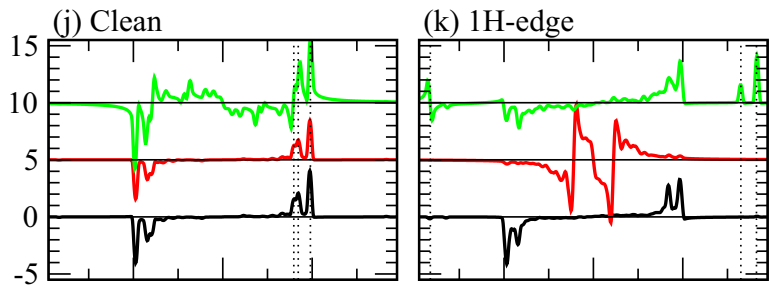

(1) $8 \mathrm{H}$-free

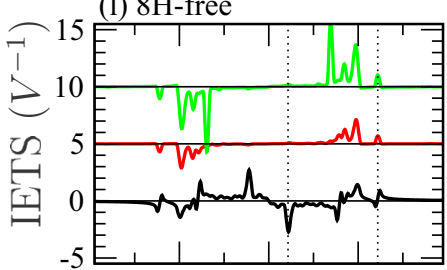

(m) 1C-broken

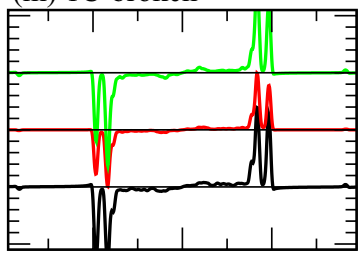

(n) 4C-broken

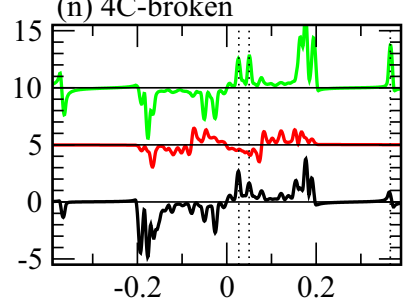

(o) Cu-adatom

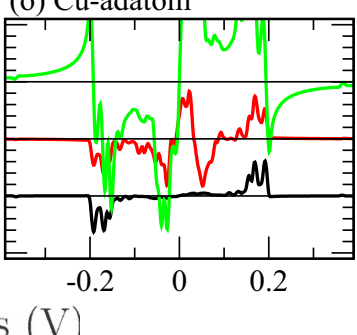

FIG. 10. (Color online) (a)-(i) IETS as a function of gate voltage $V_{G}$ for the pristine and defective AGNR structures shown in Fig. 8. (j)-(o) IETS for six selected structures at three specific gate values [dashed horizontal lines in (a)-(i)]. The curves are offset with the most negative gate value at the bottom (black curves) and the most positive at the top (green curves). (j) Clean AGNR at gate values $V_{G}=-0.3$, 0.0 , and $0.8 \mathrm{~V}$. (k) $1 \mathrm{H}$-edge at $V_{G}=-0.3,0.0$, and $0.2 \mathrm{~V}$. (1) $8 \mathrm{H}$-free at $V_{G}=-0.3,0.0$, and $0.6 \mathrm{~V}$. (m) $1 \mathrm{C}$-broken at $V_{G}=-0.3,0.0$, and $0.3 \mathrm{~V}$. (n) $4 \mathrm{C}$-broken at $V_{G}=-0.3,0.0$, and $0.3 \mathrm{~V}$. (o) Cu-adatom at $V_{G}=-0.3,0.0$, and $0.3 \mathrm{~V}$. Dotted vertical lines are guides to the eye of characteristic IETS signals corresponding to the modes in Fig. 11.

the transport characteristics with the different types of defects in AGNRs.

\section{Edge passivation}

Considering defects in the edge passivation [Figs. 8(b)8(e)] the gap in the transmission is essentially unchanged [Figs. 9(b)-9(e)], except for the 1H-edge structure where a zero-energy resonance appears in the DOS and transmission [Fig. 9(b)]. This new peak can be attributed to tunneling via a midgap state which appears due to the local breaking of sublattice symmetry [1]. Thus, if a $\mathrm{H}$ atom is added to the neighboring $\mathrm{C}$ atom [2H-edge, Fig. 8(c)] the peak disappears [Fig. 9(c)]. The addition of one or two $\mathrm{H}$ atoms on the same side also results in the closing of one transmission channel between the valence and conduction bands as shown in Figs. 9(b) and 9(c). Concerning the vibrational degrees of freedom, the addition of extra hydrogen to the edge results in new vibrational modes around $330 \mathrm{meV}$ for $1 \mathrm{H}$-edge and around 343 and $353 \mathrm{meV}$ for $2 \mathrm{H}$-edge, clearly outside the bulk phonon band (ranging up to $\sim 200 \mathrm{meV}$ ) of pristine AGNR [56].

Comparing the IETS in Figs. 10(a)-10(c) we find that only $1 \mathrm{H}$-edge gives a signal which differs significantly from the pristine case. Figure 10(k) shows specific IETS for selected gate voltages for $1 \mathrm{H}$-edge. Here at $V_{G}=0.2 \mathrm{~V}$ (top green curve) we see how new signals appear at large voltages: For positive bias polarity two signals appear at 330 and $365 \mathrm{meV}$, respectively, while for negative bias polarity only an asymmetric signal around $-365 \mathrm{meV}$ is present. The signal at $330 \mathrm{meV}$ is due to vibrations of the $\mathrm{H}_{2}$ [Fig. 11(b)], while the signal at $365 \mathrm{meV}$ [Fig. 11(a)] is due to the $\mathrm{H}$ atom on the neighboring $\mathrm{C}$ atom. Furthermore, the amplitude of the signals around 169 and $196 \mathrm{meV}$ is also found to depend on bias polarity.

Gating onto the zero-energy resonance for $1 \mathrm{H}$-edge the IETS signal [middle red curve in Fig. 10(k)] is dominated by large asymmetric signals for low energy vibrations due to the contribution from $\kappa_{\lambda}$ and Eq. (4). We note that $\kappa_{\lambda}$ changes sign with bias polarity for this approximately left-right symmetric structure. This can be seen from the red IETS curve in Fig. 10(k) which is roughly an odd function of the bias voltage. In close proximity of the zero-energy resonance a characteristic "X shape" is observed in the gate-dependent IETS, while away from it the signals approach that of the pristine AGNR [Fig. 10(b)].

Substituting a $\mathrm{H}$ atom with a $\mathrm{F}$ atom ( $1 \mathrm{~F}$-edge) is seen to have virtually no effect in the IETS of Fig. 10(d). This suggests that a significant change in the chemical composition directly 


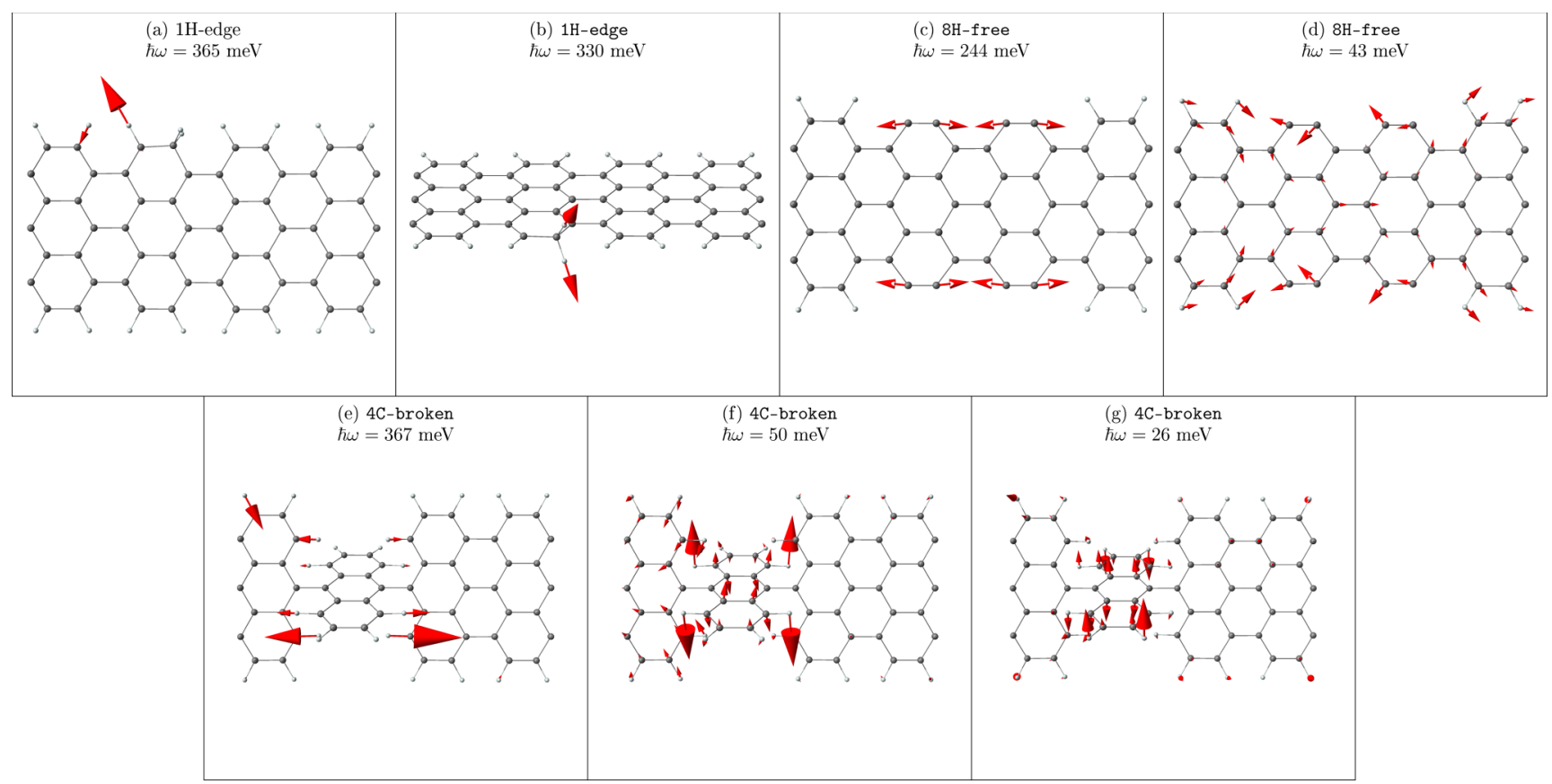

FIG. 11. (Color online) Visualization of the most contributing defect-induced vibrational modes to the IETS signals indicated by vertical lines in Figs. 10(j)-10(o). (a) and (b) The two hydrogen signals for $1 \mathrm{H}$-edge. (c) Localized edge mode at the carbon dimers for the $8 \mathrm{H}-\mathrm{free}$. (d) Delocalized edge mode for the 8H-free. (e) Hydrogen mode from the zigzag edge of 4C-broken. (f) and (g) Defect modes for 4C-broken.

involving the $\pi$-electronic system is required in order to obtain a signal although the vibrations are influenced by the heavier passivation.

Such a significant change in the passivation occurs for instance by removing four $\mathrm{H}$ atoms on each side (8H-free), giving rise to four very narrow peaks in the DOS around the conduction band [Fig. 9(e)]. These correspond to very localized dangling-bond states on the dehydrogenated dimers and therefore do not show up in the transmission. However, the dehydrogenated edges give rise to localized vibrations outside the range of the pristine vibrational spectrum [56]. The in-phase vibration of the dehydrogenated $\mathrm{C}$ dimers at the armchair edges [Fig. 11(f)] gives rise to an extra IETS peak at $244 \mathrm{meV}$ [Fig. 10(1)] matching the $\mathrm{H}$-free mode measured by Raman [34]. We find that this signal is robust as it appears in the whole range of gate values. When gating into to the valence band a new signal appears around $43 \mathrm{meV}\left[V_{G} \approx-0.8 \mathrm{~V}\right.$ in Fig. 10(e)] originating from a low energy edge vibration [Fig. 11(g)].

\section{Structural defects}

The electronic transmission in GNRs is mediated by the carbon $\pi$ system. Thus if a $\mathrm{C}-\mathrm{C}$ bond fails to be formed during GNR synthesis or if it is broken again at a later stage, a large effect can be expected for the electronic conduction properties. This impact is indeed revealed in Figs. 9(f)-9(h). Breaking one or two bonds results in the formation of two in-gap states which, broadened by the electrodes, make the gap appear smaller. The IETS signals for the $1 \mathrm{C}$-broken and 2C-broken in Figs. 10(f), 10(g), and 10(m) have the same two signals at 169 and $196 \mathrm{meV}$ as for the clean ribbon. However, the relative amplitudes are interchanged such that the $D$ peak is now slightly more intense than the $G$ peak.
Breaking four C-C bonds [4C-broken, Fig. 8(h)], resulting in constrictions of single $\mathrm{C}-\mathrm{C}$ bonds, totally alter the DOS which is now dominated by three sharp peaks as seen in Fig. 9(h). The corresponding IETS signals are shown in Figs. 10(h) and 10(n). In the proximity of the zero-energy resonance a broad range of signals at low vibrational energies appears [red curve in Fig. 10(n)] as well as a characteristic X shape in the gate plot [Fig. 10(h)] similar to that of $1 \mathrm{H}$-edge. Gating away from the resonance we observe two additional robust IETS signals at 27 and $50 \mathrm{meV}$ resulting from vibrations localized at the defect [Figs. 11(d) and 11(e)].

\section{Adatom}

Transition metals are typically used for growth of graphene or as a substrate for the bottom-up synthesis of GNRs. Thus it is of interest to consider the effect of adatoms of this type on GNRs. A Cu adatom on graphene adsorbs preferentially in the on-top position [57]. However, positioning $\mathrm{Cu}$ such that it breaks the axial symmetry of our AGNR, we find that it is most stable in a hollow site at the edge [Cu-adatom, Fig. 8(i)]. The DOS and transmission in Fig. 9(i) reveal as $n$-type doping effect shifting $E_{F}$ close to the conduction band while leaving the two transmission channels inside the gap relatively intact.

For the pristine GNR the e-ph couplings of the out-ofplane vibrations are suppressed due to the symmetry of the $\pi$ orbitals. However, around the onset of the conduction band the IETS signals in Figs. 10(i) and 10(o) are dominated by large asymmetric signals with significant contributions from out-of-plane phonons. These modes come into play due to breaking of the planar symmetry by the adatom. Also note that by gating of $E_{F}$ within the gap these signatures of the adatom disappear, cf. the lower black curve in Fig. 10(o). 


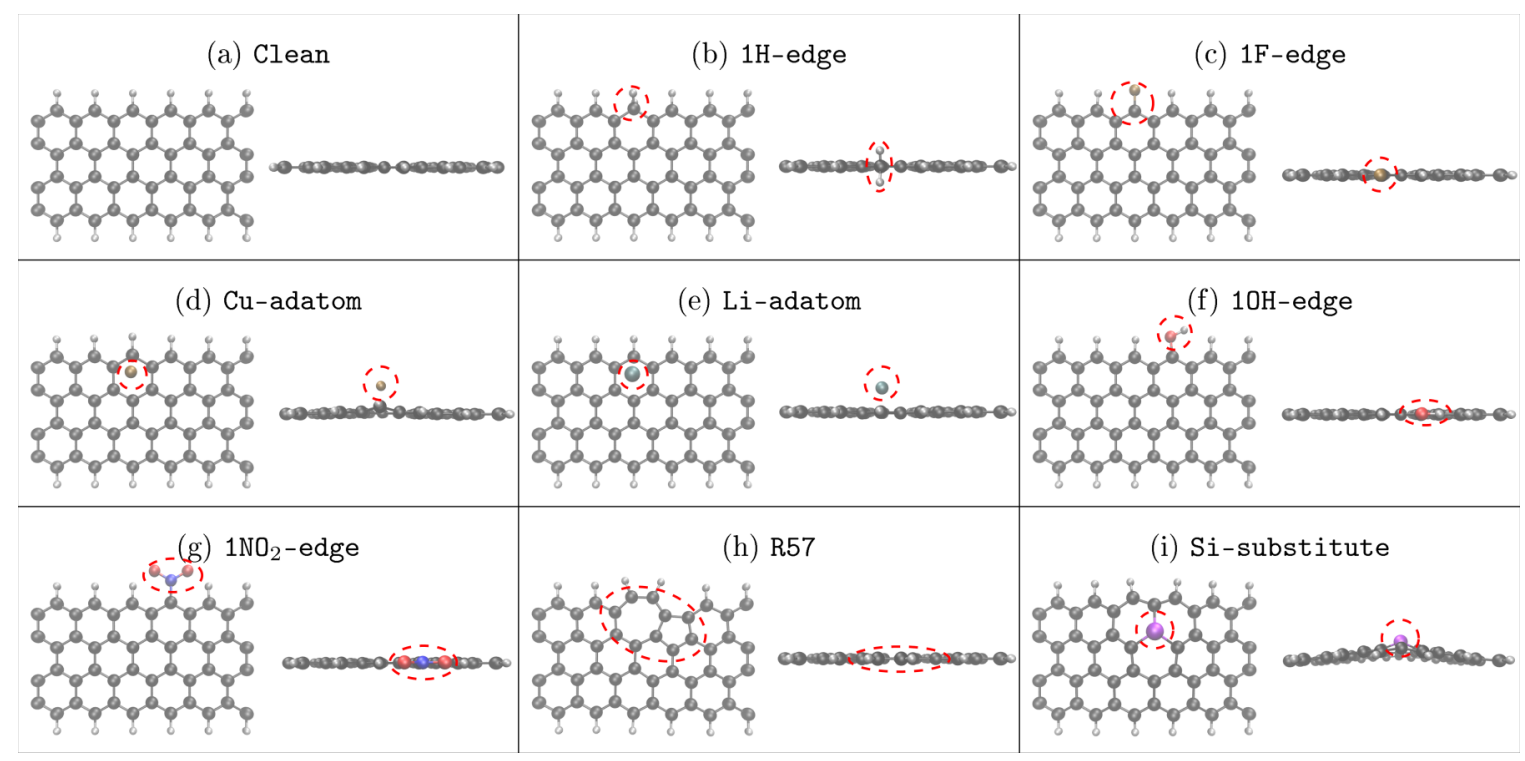

FIG. 12. (Color online) Top and side views of the dynamical region describing the various ZGNR defect structures. The dashed red ellipses are guides to the eye highlighting the defect position. (a) Pristine ZGNR. (b) One extra H atom on one of the edges. (c) One H atom replaced by a $\mathrm{F}$ atom. (d) $\mathrm{Cu}$ adatom in a hollow site on the edge. (e) Li adatom in a hollow site on the edge. (f) One $\mathrm{H}$ replaced by a OH group. (g) One $\mathrm{H}$ replaced by a $\mathrm{NO}_{2}$ group. (h) Structural defect (R57). (i) Substitutional Si defect next to the edge.

\section{B. Defects in ZGNRs}

Let us next consider a series of defects for the zigzag graphene nanoribbon. Due to the different size and orientation of the AGNR and ZGNR unit cell their structural defects will generally differ. Thus, instead of the range of structural defects considered for the AGNR we investigate a number of chemical defects, both as adatoms, in the edge passivation and substituting a carbon atom. Lithium binding to carbon is of relevance to carbon-based Li-ion batteries [58] and DFT studies have shown edge-oxidized zigzag graphene to be more stable than hydrogen terminated $[6,25]$, furthermore, $\mathrm{NO}_{2}$ groups were shown to be Raman active [59]. Thus in Fig. 12 we show the atomic structures of pristine ZGNR along with eight different defect configurations. We consider the following defects in the edge passivation: A single edge with an extra hydrogen [1H-edge, Fig. 12(b)], one hydrogen is replaced by either a $\mathrm{F}$ atom [1F-edge, Fig. 12(c)], an $\mathrm{OH}$ group [10H-edge, Fig. 12(f)], or a $\mathrm{NO}_{2}$ group [ $1 \mathrm{NO}_{2}$-edge, Fig. $12(\mathrm{~g})]$. We also consider defects in the form of a $\mathrm{Cu}$ adatom [Cu-adatom, Fig. 12(d)] or a Li adatom [Li-adatom, Fig. 12(e)]. Finally, we also study the effect of a structural defect in form of a 57 reconstruction [R57, Fig. 12(h)] and a substitutional defect where a $\mathrm{C}$ atom next to the edge is replaced by a $\mathrm{Si}$ atom [Si-substitute, Fig. 12(i)]. For all these systems the entire dynamical region was relaxed, i.e., the parts of the ZGNRs shown in Fig. 12 using spinpolarized treatments. The spin degrees of freedom $\sigma=\uparrow, \downarrow$ generalizes $\gamma_{\lambda}^{\sigma}$ and $\kappa_{\lambda}^{\sigma}$ [Eqs. (5) and (6)] corresponding to two independent spin channels, which in general can have quite different amplitudes and even opposite sign. The observable IETS would simply be the sum of these two components $\left(\partial_{V}^{2} I_{\uparrow}+\partial_{V}^{2} I_{\downarrow}\right) /\left(\partial_{V} I_{\uparrow}+\partial_{V} I_{\downarrow}\right)$.

Similar to the AGNR case, the electronic properties in the device region with the different impurity configurations for the ZGNR, now spin resolved, are summarized in Fig. 13. The IETS of pristine ZGNR was already discussed in Sec. III B and below we continue describing the IETS fingerprints for the various defects.

\section{Edge passivation}

As commented above, the broader IETS signals of pristine ZGNR (Figs. 3 and 4) (as compared with AGNR) can be understood from the breaking of the axial mirror symmetry and hence fewer symmetry-forbidden inelastic transitions. These broader signals may in general make the detection of defect signatures more difficult. For 1H-edge [Fig. 13(b)] the IETS resembles that of the pristine ZGNR [Fig. 13(a)] inside the gap. However, gating into the valance band [black curve in Fig. 13(k)] the edge states start to extend into the middle of the ribbon, partially restoring mirror symmetry, and thus resulting in part of the pristine ZGNR signals to disappear.

Here an extra signal appear due to edge modes in the frequency range 194 to $199 \mathrm{meV}$ with the most contributing mode at $196 \mathrm{meV}$ [as shown in Fig. 15(a)]. The resulting IETS signal can clearly be seen in the bottom curve in Fig. 14(k). As for the AGNR substituting a hydrogen with a fluorine atom has a very limited effect on the electronic properties and the IETS signal.

Substituting a hydrogen with an $\mathrm{OH}$ group, according to Figs. 13(f) and 13(o), has only a small effect on the spin-down electrons, while it shrinks and add additional structure to the gap for the spin-up electrons. For the spin-up electrons there is a small peak inside the gap which gives rise to a large asymmetric IETS signal around $V_{G}=-0.2 \mathrm{~V}$ in Fig. 13(o) lower curve, compared to the pristine case. The most contributing mode to the asymmetric IETS signal is shown in Fig. 15(b). However, there is no clear signature of the $\mathrm{OH}$ 
(a) Clean

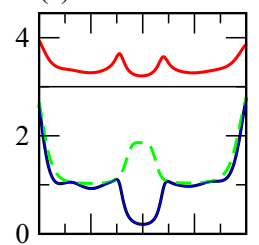

(d) Cu-adatom

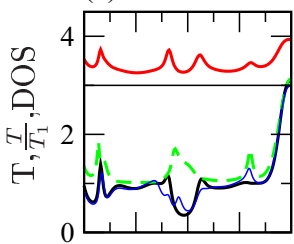

(g) $1 \mathrm{NO}_{2}$-edge

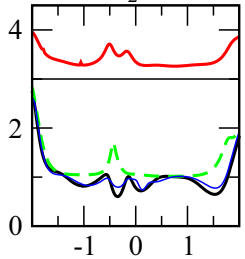

Spin up

(b) $1 \mathrm{H}$-edge

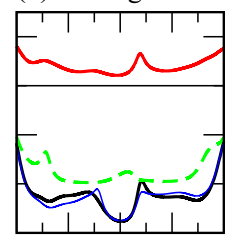

(e) Li-adatom

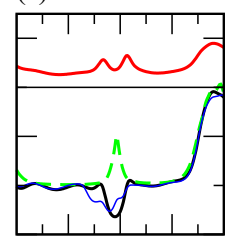

(h) R57

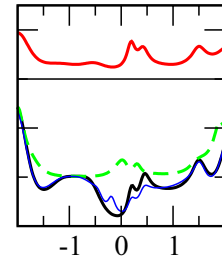

(c) $1 \mathrm{~F}$-edge

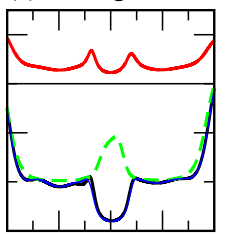

(f) $1 \mathrm{OH}$-edge

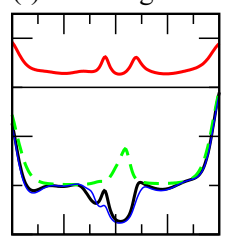

(i) Si-substitute

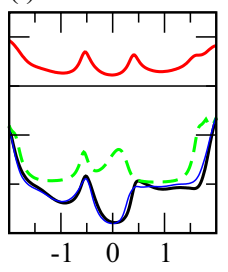

Spin down

(k) $1 \mathrm{H}$-edge

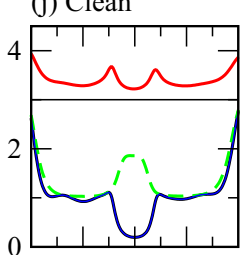

(m) Cu-adatom

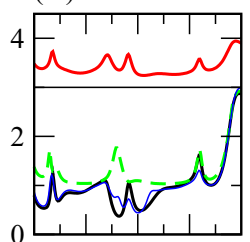

(p) $1 \mathrm{NO}_{2}$-edge

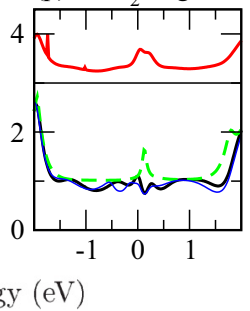

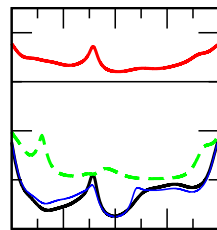

(n) Li-adatom

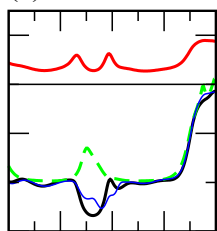

(q) R57

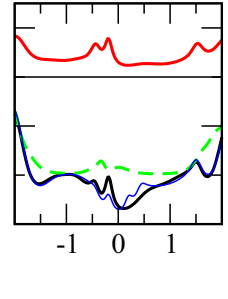

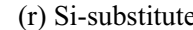

(1) 1F-edge

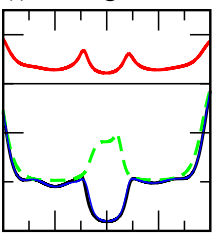

(o) $1 \mathrm{OH}$-edge

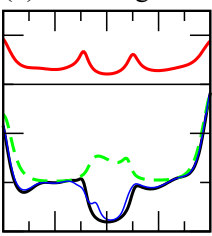

(r) Si-substitute

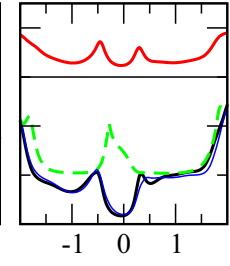

FIG. 13. (Color online) Electronic properties of the ZGNR structures shown in Fig. 12 with the spin-up/down components in the left/right panel. The spin-resolved total transmission is shown with black lines while spin-averaged total transmission is shown with thin blue lines. The ratio $T^{\sigma} / T_{1}^{\sigma}$, where $T_{1}^{\sigma}$ is the transmission originating from the most transmitting spin eigenchannel, is shown with green dashed lines (this ratio gives a lower bound to the number of contributing eigenchannels with spin $\sigma$ ). The spin-resolved DOS (arb. units) for the $\mathrm{C}$ atoms in the dynamical region is shown with red lines (offset by three units).

group itself. In the same manner the substitution with a $\mathrm{NO}_{2}$ group removes the gap in the electronic properties without leaving any direct fingerprint of the $\mathrm{NO}_{2}$ group in the IETS signal.

\section{Adatom}

As for the AGNR we consider the effect of adatoms. For the $\mathrm{Cu}$ adatom the transport gap shrinks for the spin-up electrons while there is an in-gap peak for the spin-down electrons, cf. Fig. 13(m). Thus, for some gate values the IETS signals reflect that the spin-down electrons will back scatter while the spin-up electrons will be forward scattered, and the observed signal is then the sum of these contributions. For a gate value of $V_{G}=-0.2 \mathrm{~V}$, the IETS signal is dominated by spin-down electrons. Due to the finite width of the in-gap peak, in the spin-down transmission, the low frequency phonons $(\hbar \omega<$ $0.1 \mathrm{meV}$ ) give rise to back scattering while the high frequency phonons $(\hbar \omega>0.1 \mathrm{meV})$ result in forward scattering. Thus, the low and high energy signals have different signs as can be seen from Fig. 14(1). Interestingly, the low energy signal primarily consists of symmetric contributions from out-ofplane modes [Fig. 15(c)]. Replacing the $\mathrm{Cu}$ adatom with $\mathrm{Li}$, the transmission and DOS, shown in Figs. 13(e) and 13(n), reveals a spin dependent $n$-type doping effect, where $E_{F}$ is shifted the most for spin-down. However, no in-gap peak is seen as for $\mathrm{Cu}$ and the IETS show no clear signature of the $\mathrm{Li}$ atom.

\section{Structural defect}

The formation of a $\mathrm{R} 57$ reconstruction results in peaks in the DOS in the device region, just above $E_{F}$ for spin-up [Fig. 13(h)] and just below $E_{F}$ for spin-down [Fig. 13(q)]. The R57 breaks the symmetry both in the vibrational and electronic structure allowing for IETS signals from a wider range of vibrations, resulting in broader peaks, as seen from Figs. 14(h) and 14(n). One of the contributing modes is localized at the border between the pentagon ring and middle of the ribbon at $\hbar \omega=204 \mathrm{meV}$ [Fig. 15(d)]. This localized mode yields a relatively small signal compared to the other signals, however, contrary to the other modes the localized mode is not expected to be broadened if the coupling to phonons away from the dynamical region is taken into account. The breaking of symmetry in the electronic structure also give rise to difference signals for the two bias polarities.

\section{Substitutional impurity}

Substituting a carbon with a silicon atom leads to an out-of-plane buckling, see Fig. 12(i). However, both silicon and carbon have an $s^{2} p^{2}$ electronic structure, and the electron transmission is basically similar to the pristine. On the other hand, the buckling gives rise to low energy peaks in the IETS signal originating from the e-ph coupling to the out-of-plane modes [Fig. 15(e)]. Gating close to the band edge of the conduction band gives rise to different sign of the signals at low and high vibrational energies, as seen from the top curve in Fig. 14(o). 


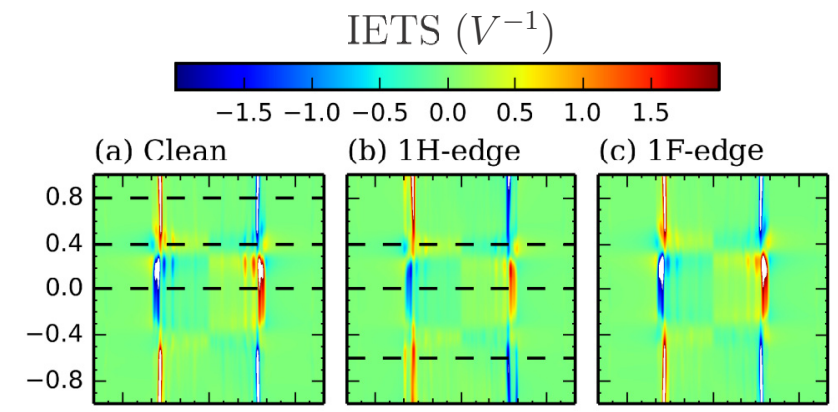

(d) Cu-adatom

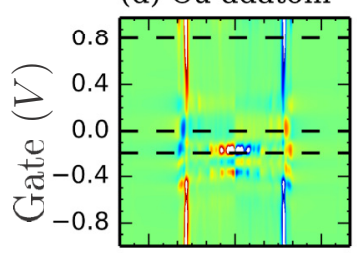

(g) $1 \mathrm{NO} 2$-edge

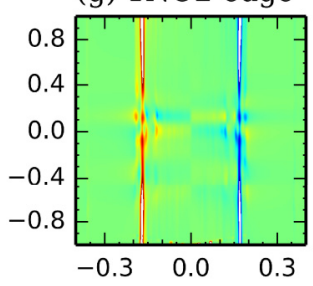

(e) Li-adatom

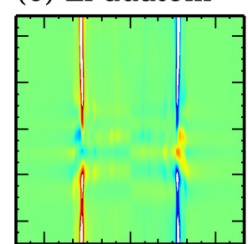

(h) R57

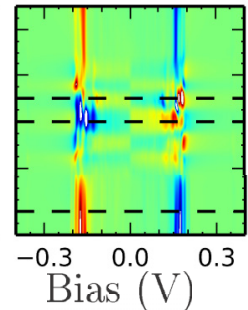

(f) $10 \mathrm{OH}$-edge

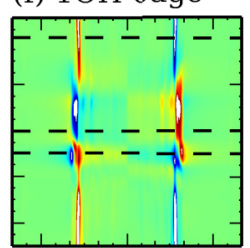

(i) Si-substitute

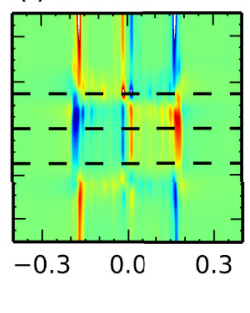

(j) Clean

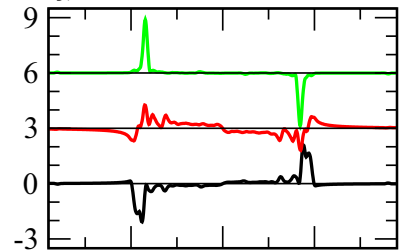

(1) $\mathrm{Cu}$-adatom

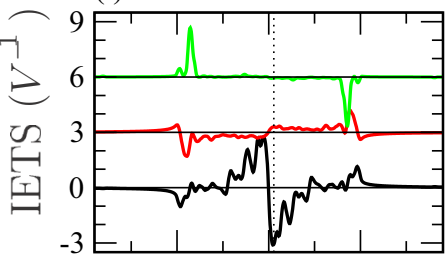

(n) R57

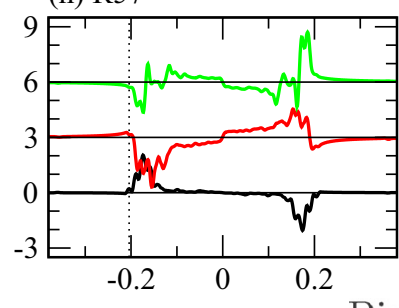

(k) 1H-edge

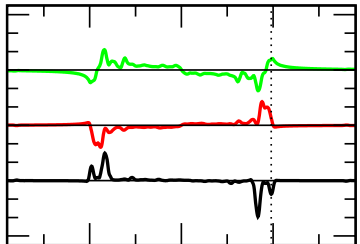

(m) 1OH-edge

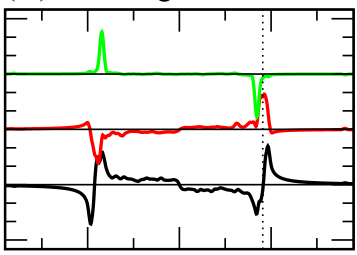

(o) Si-substitute

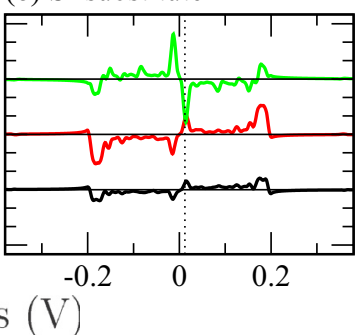

FIG. 14. (Color online) (a)-(i) Spin-averaged IETS as a function of gate voltage $V_{G}$ for the pristine and defective ZGNR structures shown in Fig. 12. (j)-(o) IETS for six selected structures at three specific gate values [dashed horizontal lines in (a)-(i)]. The curves are offset with the most negative gate value at the bottom (black curves) and the most positive at the top (green curves). (j) Clean ZGNR for gate values $V_{G}=0.0$, 0.4 , and $0.8 \mathrm{~V}$. (k) $1 \mathrm{H}$-edge at $V_{G}=-0.6,0.0$, and $0.4 \mathrm{~V}$. (l) Cu-adatom at $V_{G}=-0.2,0.0$, and $0.8 \mathrm{~V}$. (m) $10 \mathrm{H}-$ edge at $V_{G}=-0.2,0.0$, and $0.8 \mathrm{~V}$. (n) R57 at $V_{G}=-0.8,0.0$, and $0.2 \mathrm{~V}$. (o) Si-substitute at $V_{G}=-0.3,0.0$, and $0.3 \mathrm{~V}$. Dotted vertical lines are guides to the eye of characteristic IETS signals corresponding to the modes shown in Fig. 15.

\section{CONCLUSIONS}

In summary, we have investigated IETS signals in symmetrically contacted armchair and zigzag graphene nanoribbons, considering both pristine as well as a selection of defective configurations under varying charge carrier conditions. For the clean AGNR inelastic tunneling gives rise to two distinct peaks in the IETS spectrum at 169 and $196 \mathrm{mV}$ corresponding to the $D$ and $G$ modes of Raman spectroscopy, respectively. By connecting the IETS signals to the phonon band structure, we have clarified how only a single band contributes to the $G$ mode while three bands contribute to the broader $D$ mode. Concerning defects in AGNRs we have shown how some leave IETS unchanged while others give clear signals. For instance, adding an extra hydrogen atom to a single edge side gives a clear signal for some gate values. This signal can be removed by adding another hydrogen atom to the neighboring edge side because the sublattice symmetry is restored. Furthermore, exchanging a single hydrogen atom with a fluorine atom in the passivation does not result in any change in both the elastic and inelastic tunneling. However, removing eight hydrogen atoms leaving part of the edge on each side without passivation, gives a clear robust signal throughout the investigated gate values. The signal, due to the vibration of the carbon dimers at the edge, has an energy around $245 \mathrm{meV}$ making it easy to detect since it is outside the vibrational spectrum of the pristine ribbon. Breaking of one or two $\mathrm{C}-\mathrm{C}$ bonds turns out to interchange the relative intensity of the $G$ and $D$ peaks. Breaking four $\mathrm{C}-\mathrm{C}$ bonds gives rise to signals caused by the defect tilted out-of-plane. Lifting the symmetry of the $\pi$ electrons by adding a $\mathrm{Cu}$ adatom allows the out-of-plane modes to contribute.

For the ZGNR we find relatively broader IETS signals especially in the absence of a large gate voltage $\left(V_{G} \approx\right.$ $0 \mathrm{~V}$ ). Importantly, this is a consequence of the breaking of the axial mirror symmetry in the ribbon due to the presence of spin-polarized edges. Thus, by comparing to spin-degenerate calculations, we suggest that IETS can give an indirect proof of spin polarization in zigzag ribbons. On the other hand, the broader IETS features may make it difficult to identify the different defect signals reported in this paper.

The presence of a $\mathrm{R} 57$ reconstruction also broadens the IETS by breaking both the electronic and vibrational symmetry. Substituting a carbon atom with a silicon atom makes the ribbon buckle, breaking the planar symmetry, allowing the 


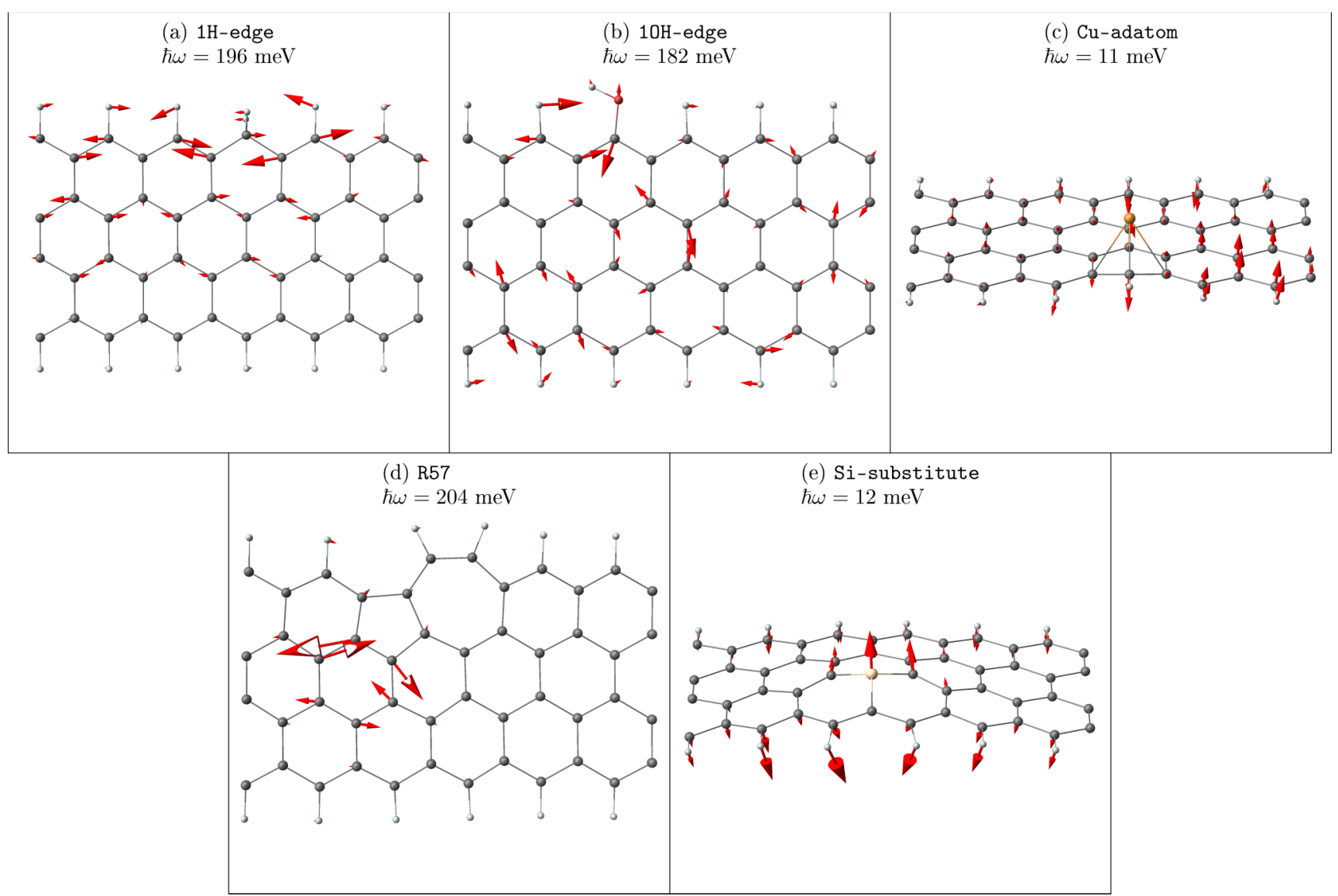

FIG. 15. (Color online) Visualization of the most contributing modes to the IETS signals indicated by vertical lines in Figs. 14(j)-14(o). (a) Edge mode at the edge with the extra hydrogen in 1H-edge. (b) Mode contributing to the asymmetric signal in Fig. 14(m) for the 10H-edge. (c) Out-of-plane mode for $\mathrm{Cu}$-adatom. (d) Localized mode for R57. (e) Out-of-plane mode for Si-substitute.

out-of-plane modes to contribute to the IETS. This suggests that IETS in principle could be used to gain information of the curvature of GNRs and other graphene-based structures.

Finally, as an outlook we note that here we presented calculations on long, symmetrically contacted systems where there is a significant overlap with both metallic electrodes. It would be interesting to extend such a study also to the asymmetric situation where a point tunnel contact is made to one end resembling, say, the coupling to a STM tip [20].

\section{ACKNOWLEDGMENTS}

T.F. thanks the Center for Nanostructured Graphene (Project DNRF58) for hospitality during a visit supported by the Villum Foundation and acknowledges support from the Basque Departamento de Educación and the UPV/EHU (Grant No. IT-756-13), the Spanish Ministerio de Economía y Competitividad (Grant No. MAT2013-46593-C6-2-P), and the European Union FP7-ICT project PAMS (Contract No. 610446).
[1] H. Raza, Graphene Nanoelectronics: Metrology, Synthesis, Properties and Applications (Springer, New York, 2011).

[2] L. E. F. Foa Torres, S. Roche, and J.-C. Charlier, Introduction to Graphene-Based Nanomaterials: From Electronic Structure to Quantum Transport (Cambridge University Press, Cambridge, 2014).

[3] S. Dutta and S. K. Pati, Novel properties of graphene nanoribbons: A review, J. Mater. Chem. 20, 8207 (2010).

[4] T. G. Pedersen, C. Flindt, J. Pedersen, N. A. Mortensen, A.-P. Jauho, and K. Pedersen, Graphene antidot lattices: Designed defects and spin qubits, Phys. Rev. Lett. 100, 136804 (2008).
[5] J. Bai, X. Zhong, S. Jiang, Y. Huang, and X. Duan, Graphene nanomesh, Nat. Nanotechnol. 5, 190 (2010).

[6] P. Wagner, C. P. Ewels, J.-J. Adjizian, L. Magaud, P. Pochet, S. Roche, A. Lopez-Bezanilla, V. V. Ivanovskaya, A. Yaya, M. Rayson et al., Band gap engineering via edge-functionalization of graphene nanoribbons, J. Phys. Chem. C 117, 26790 (2013).

[7] A. C. Ferrari and D. M. Basko, Raman spectroscopy as a versatile tool for studying the properties of graphene, Nat. Nanotechnol. 8, 235 (2013).

[8] A. Shiotari, T. Kumagai, and M. Wolf, Tip-enhanced Raman spectroscopy of graphene nanoribbons on $\mathrm{Au}(111)$, J. Phys. Chem. C 118, 11806 (2014). 
[9] M. Y. Han, B. Özyilmaz, Y. Zhang, and P. Kim, Energy bandgap engineering of graphene nanoribbons, Phys. Rev. Lett. 98, 206805 (2007).

[10] X. Li, X. Wang, L. Zhang, S. Lee, and H. Dai, Chemically derived, ultrasmooth graphene nanoribbon semiconductors, Science 319, 1229 (2008).

[11] Z.-S. Wu, W. Ren, L. Gao, B. Liu, J. Zhao, and H.-M. Cheng, Efficient synthesis of graphene nanoribbons sonochemically cut from graphene sheets, Nano Res. 3, 16 (2010).

[12] J. Baringhaus, M. Ruan, F. Edler, A. Tejeda, M. Sicot, A. Taleb-Ibrahimi, A.-P. Li, Z. Jiang, E. H. Conrad, C. Berger et al., Exceptional ballistic transport in epitaxial graphene nanoribbons, Nature (London) 506, 349 (2014).

[13] D. V. Kosynkin, A. L. Higginbotham, A. Sinitskii, J. R. Lomeda, A. Dimiev, B. K. Price, and J. M. Tour, Longitudinal unzipping of carbon nanotubes to form graphene nanoribbons, Nature (London) 458, 872 (2009).

[14] J. Cai, P. Ruffieux, R. Jaafar, M. Bieri, T. Braun, S. Blankenburg, M. Muoth, A. P. Seitsonen, M. Saleh, X. Feng et al., Atomically precise bottom-up fabrication of graphene nanoribbons, Nature (London) 466, 470 (2010).

[15] S. Blankenburg, J. Cai, P. Ruffieux, R. Jaafar, D. Passerone, X. Feng, K. Müllen, R. Fasel, and C. A. Pignedoli, Intraribbon heterojunction formation in ultranarrow graphene nanoribbons, ACS Nano 6, 2020 (2012).

[16] P. Han, K. Akagi, F. Federici Canova, H. Mutoh, S. Shiraki, K. Iwaya, P. S. Weiss, N. Asao, and T. Hitosugi, Bottomup graphene-nanoribbon fabrication reveals chiral edges and enantioselectivity, ACS Nano 8, 9181 (2014).

[17] J. Cai, C. A. Pignedoli, L. Talirz, P. Ruffieux, H. Söde, L. Liang, V. Meunier, R. Berger, R. Li, X. Feng et al., Graphene nanoribbon heterojunctions, Nat. Nanotechnol. 9, 896 (2014).

[18] C. Bronner, F. Leyssner, S. Stremlau, M. Utecht, P. Saalfrank, T. Klamroth, and P. Tegeder, Electronic structure of a subnanometer wide bottom-up fabricated graphene nanoribbon: End states, band gap, and dispersion, Phys. Rev. B 86, 085444 (2012).

[19] P. Ruffieux, J. Cai, N. C. Plumb, L. Patthey, D. Prezzi, A. Ferretti, E. Molinari, X. Feng, K. Müllen, C. A. Pignedoli et al., Electronic structure of atomically precise graphene nanoribbons, ACS Nano 6, 6930 (2012).

[20] J. van der Lit, M. P. Boneschanscher, D. Vanmaekelbergh, M. Ijäs, A. Uppstu, M. Ervasti, A. Harju, P. Liljeroth, and I. Swart, Suppression of electron-vibron coupling in graphene nanoribbons contacted via a single atom, Nat. Commun. 4, 2023 (2013).

[21] M. Koch, F. Ample, C. Joachim, and L. Grill, Voltage-dependent conductance of a single graphene nanoribbon, Nat. Nanotechnol. 7, 713 (2012).

[22] T. Wassmann, A. P. Seitsonen, A. M. Saitta, M. Lazzeri, and F. Mauri, Structure, stability, edge states, and aromaticity of graphene ribbons, Phys. Rev. Lett. 101, 096402 (2008).

[23] J. Li, Z. Li, G. Zhou, Z. Liu, J. Wu, B.-L. Gu, J. Ihm, and W. Duan, Spontaneous edge-defect formation and defectinduced conductance suppression in graphene nanoribbons, Phys. Rev. B 82, 115410 (2010).

[24] P. Wagner, V. V. Ivanovskaya, M. Melle-Franco, B. Humbert, J.-J. Adjizian, P. R. Briddon, and C. P. Ewels, Stable hydrogenated graphene edge types: Normal and reconstructed Klein edges, Phys. Rev. B 88, 094106 (2013).
[25] O. Hod, V. Barone, J. E. Peralta, and G. E. Scuseria, Enhanced half-metallicity in edge-oxidized zigzag graphene nanoribbons, Nano Lett. 7, 2295 (2007).

[26] D. Gunlycke, J. Li, J. W. Mintmire, and C. T. White, Altering low-bias transport in zigzag-edge graphene nanostrips with edge chemistry, Appl. Phys. Lett. 91, 112108 (2007).

[27] C. Zhang, C. He, L. Xue, K. Zhang, L. Sun, and J. Zhong, Transport properties of zigzag graphene nanoribbons with oxygen edge decoration, Org. Electron. 13, 2494 (2012).

[28] N. Al-Aqtash, H. Li, L. Wang, W.-N. Mei, and R. Sabirianov, Electromechanical switching in graphene nanoribbons, Carbon 51, 102 (2013).

[29] T. Yamamoto, K. Watanabe, and K. Mii, Empirical-potential study of phonon transport in graphitic ribbons, Phys. Rev. B 70, 245402 (2004).

[30] M. Vandescuren, P. Hermet, V. Meunier, L. Henrard, and P. Lambin, Theoretical study of the vibrational edge modes in graphene nanoribbons, Phys. Rev. B 78, 195401 (2008).

[31] R. Gillen, M. Mohr, J. Maultzsch, and C. Thomsen, Lattice vibrations in graphene nanoribbons from density functional theory, Phys. Status Solidi 246, 2577 (2009).

[32] J. Zhou and J. Dong, Vibrational property and Raman spectrum of carbon nanoribbon, Appl. Phys. Lett. 91, 173108 (2007).

[33] R. Saito, M. Furukawa, G. Dresselhaus, and M. S. Dresselhaus, Raman spectra of graphene ribbons, J. Phys. Condens. Matter 22, 334203 (2010).

[34] H. Huang, D. Wei, J. Sun, S. L. Wong, Y. P. Feng, A. H. C. Neto, and A. T. S. Wee, Spatially resolved electronic structures of atomically precise armchair graphene nanoribbons, Sci. Rep. 2, 983 (2012).

[35] M. Ijäs, M. Ervasti, A. Uppstu, P. Liljeroth, J. van der Lit, I. Swart, and A. Harju, Electronic states in finite graphene nanoribbons: Effect of charging and defects, Phys. Rev. B 88, 075429 (2013).

[36] J.-T. Lü, R. B. Christensen, G. Foti, T. Frederiksen, T. Gunst, and M. Brandbyge, Efficient calculation of inelastic vibration signals in electron transport: Beyond the wide-band approximation, Phys. Rev. B 89, 081405 (2014).

[37] J. M. Soler, E. Artacho, J. D. Gale, A. García, J. Junquera, P. Ordejón, and D. Sánchez-Portal, The SIESTA method for $a b$ initio order- $N$ materials simulation, J. Phys. Condens. Matter 14, 2745 (2002).

[38] M. Brandbyge, J.-L. Mozos, P. Ordejón, J. Taylor, and K. Stokbro, Density-functional method for nonequilibrium electron transport, Phys. Rev. B 65, 165401 (2002).

[39] J. P. Perdew, K. Burke, and M. Ernzerhof, Generalized gradient approximation made simple, Phys. Rev. Lett. 77, 3865 (1996).

[40] M. Paulsson, T. Frederiksen, and M. Brandbyge, Modeling inelastic phonon scattering in atomic- and molecular-wire junctions, Phys. Rev. B 72, 201101 (2005).

[41] T. Frederiksen, M. Paulsson, M. Brandbyge, and A.-P. Jauho, Inelastic transport theory from first principles: Methodology and application to nanoscale devices, Phys. Rev. B 75, 205413 (2007).

[42] http://sourceforge.net/projects/inelastica.

[43] M. P. L. Sancho, J. M. L. Sancho, and J. Rubio, Quick iterative scheme for the calculation of transfer matrices: Application to Mo (100), J. Phys. F 14, 1205 (1984). 
[44] M. Paulsson and M. Brandbyge, Transmission eigenchannels from nonequilibrium Green's functions, Phys. Rev. B 76, 115117 (2007).

[45] C. Bronner, M. Utecht, A. Haase, P. Saalfrank, T. Klamroth, and $\mathrm{P}$. Tegeder, Electronic structure changes during the surfaceassisted formation of a graphene nanoribbon, J. Chem. Phys. 140, 024701 (2014).

[46] L. Yang, C.-H. Park, Y.-W. Son, M. L. Cohen, and S. G. Louie, Quasiparticle energies and band gaps in graphene nanoribbons, Phys. Rev. Lett. 99, 186801 (2007).

[47] X. Jiang, N. Kharche, P. Kohl, T. B. Boykin, G. Klimeck, M. Luisier, P. M. Ajayan, and S. K. Nayak, Giant quasiparticle bandgap modulation in graphene nanoribbons supported on weakly interacting surfaces, Appl. Phys. Lett. 103, 133107 (2013).

[48] L. Liang and V. Meunier, Electronic structure of assembled graphene nanoribbons: Substrate and many-body effects, Phys. Rev. B 86, 195404 (2012).

[49] H. Song, Y. Kim, Y. H. Jang, H. Jeong, M. A. Reed, and T. Lee, Observation of molecular orbital gating, Nature (London) 462, 1039 (2009).

[50] M. Paulsson, T. Frederiksen, H. Ueba, N. Lorente, and M. Brandbyge, Unified description of inelastic propensity rules for electron transport through nanoscale junctions, Phys. Rev. Lett. 100, 226604 (2008).

[51] B. N. J. Persson and A. Baratoff, Inelastic electron-tunneling from a metal tip - the contribution from resonant processes, Phys. Rev. Lett. 59, 339 (1987).
[52] N. Lorente and M. Persson, Theory of single molecule vibrational spectroscopy and microscopy, Phys. Rev. Lett. 85, 2997 (2000).

[53] S. Kim and Y.-W. Son, Scattering theory approach to inelastic transport in nanoscale systems, Phys. Rev. B 87, 195423 (2013).

[54] D. Djukic and J. M. van Ruitenbeek, Shot noise measurements on a single molecule, Nano Lett. 6, 789 (2006).

[55] N. L. Schneider, J. T. Lü, M. Brandbyge, and R. Berndt, Light emission probing quantum shot noise and charge fluctuations at a biased molecular junction, Phys. Rev. Lett. 109, 186601 (2012).

[56] M. Engelund, J. A. Fürst, A. P. Jauho, and M. Brandbyge, Localized edge vibrations and edge reconstruction by Joule heating in graphene nanostructures, Phys. Rev. Lett. 104, 036807 (2010).

[57] X. Liu, C. Z. Wang, M. Hupalo, W. C. Lu, M. C. Tringides, Y. X. Yao, and K. M. Ho, Metals on graphene: Correlation between adatom adsorption behavior and growth morphology, Phys. Chem. Chem. Phys. 14, 9157 (2012).

[58] Y. Liu, Y. M. Wang, B. I. Yakobson, and B. C. Wood, Assessing carbon-based anodes for lithium-ion batteries: A universal description of charge-transfer binding, Phys. Rev. Lett. 113, 028304 (2014).

[59] C. M. Berg and D. D. Dlott, Picosecond dynamics of shock compressed and flash-heated nanometer thick films of $\delta$-HMX, J. Phys. Conf. Ser. 500, 142004 (2014). 